\title{
Species-dependent neuropathology in transgenic SOD1 pigs
}

Huaqiang Yang, ${ }^{1, *}$, Guohao Wang ${ }^{2,}$, , Haitao Sun ${ }^{3, *}$, Runzhe Shu, ${ }^{4, *}$, Tao Liu ${ }^{1}$, Chuan-En Wang ${ }^{5}$, Zhaoming Liu ${ }^{1}$, Yu Zhao ${ }^{1}$, Bentian Zhao ${ }^{1}$, Zhen Ouyang ${ }^{1}$, Dongshan Yang ${ }^{1}$, Jiao Huang ${ }^{1}$, Yueling Zhou ${ }^{3}$, Shihua Li $^{5}$, Xiaodan Jiang ${ }^{3}$, Zhicheng Xiao ${ }^{3,4,6}$, Xiao-Jiang $\mathrm{Li}^{2,5}$, Liangxue Lai ${ }^{1}$

${ }^{I}$ Key Laboratory of Regenerative Biology, South China Institute for Stem Cell Biology and Regenerative Medicine, Guangzhou Institutes of Biomedicine and Health, Chinese Academy of Sciences, Guangzhou, Guangdong 510530, China; ${ }^{2}$ State Key Laboratory of Molecular Developmental Biology, Institute of Genetics and Developmental Biology, Chinese Academy of Sciences, Beijing 100101, China; ${ }^{3}$ Department of Neurosurgery, The National Key Clinic Specialty, The Neurosurgery Institute of Guangdong Province, Guangdong Provincial Key Laboratory on Brain Function Repair and Regeneration, Zhujiang Hospital, Southern Medical University, Guangzhou, Guangdong 510280, China; ${ }^{4}$ Department of Anatomy and Developmental Biology, Monash University, Clayton, Victoria 3800, Australia; ${ }^{5}$ Department of Human Genetics, Emory University School of Medicine, Atlanta, GA 30322, USA; ${ }^{6}$ The Key Laboratory of Stem Cell and Regenerative Medicine, Institute of Molecular and Clinical Medicine, Kunming Medical University, Kunming, Yunnan 650500, China

Mutations in the human copper/zinc superoxide dismutase 1 (hSOD1) gene cause familial amyotrophic lateral sclerosis (ALS). It remains unknown whether large animal models of ALS mimic more pathological events seen in ALS patients via novel mechanisms. Here, we report the generation of transgenic pigs expressing mutant G93A hSOD1 and showing hind limb motor defects, which are germline transmissible, and motor neuron degeneration in dose- and age-dependent manners. Importantly, in the early disease stage, mutant hSOD1 did not form cytoplasmic inclusions, but showed nuclear accumulation and ubiquitinated nuclear aggregates, as seen in some ALS patient brains, but not in transgenic ALS mouse models. Our findings revealed that SOD1 binds PCBP1, a nuclear poly(rC) binding protein, in pig brain, but not in mouse brain, suggesting that the SOD1-PCBP1 interaction accounts for nuclear SOD1 accumulation and that species-specific targets are key to ALS pathology in large mammals and in humans.

Keywords: neurodegeneration; motor neurons; nuclear inclusions; SOD1; PCBP1

Cell Research (2014) 24:464-481. doi:10.1038/cr.2014.25; published online 28 February 2014

\section{Introduction}

Amyotrophic lateral sclerosis (ALS) is an adult-onset progressive neurodegenerative disease that involves selective death of motor neurons (MNs). Patients with ALS develop progressive loss of upper and lower motor neu-

\footnotetext{
*These four authors contributed equally to this work.

Correspondence: Xiao-Jiang Li ${ }^{\mathrm{a}}$, Xiaodan Jiang ${ }^{\mathrm{b}}$, Zhicheng Xiao ${ }^{\mathrm{c}}$,

Liangxue Lai ${ }^{d}$

åE-mail: xli2@emory.edu

bE-mail: Jiangxiao_dan@163.com

${ }^{c}$ E-mail: zhicheng.xiao@monash.edu

${ }^{\mathrm{d}}$ E-mail: lai_liangxue@gibh.ac.cn

Received 18 August 2013; revised 18 November 2013; accepted 13 January 2014; published online 28 February 2014
}

rons, muscle atrophy, and eventually paralysis, and they usually die within 3-5 years after symptoms begin $[1,2]$. Most forms of ALS are sporadic (sALS), and $\sim 10 \%$ are familial ALS (fALS). The first mutated ALS gene discovered was the superoxide dismutase 1 (SOD1) gene, which encodes a metalloenzyme that converts the highly toxic superoxide anion to molecular oxygen and hydrogen peroxide [3]. Now over 150 pathogenic mutations in SOD1 have been found in ALS patients [4]. Identification of these mutations has led to a number of animal models expressing ALS-associated SOD1 mutants, providing strong evidence for a gain-of-toxic function of mutant SOD1 [3-6].

Mutant SOD1 transgenic rodent models are by far the most widely used animal models of ALS and have given 
us important insights into the pathogenesis of ALS and potential therapies. A variety of pathogenic mechanisms, including abnormal protein aggregation and cytoplasmic inclusion formation, excitotoxicity, mitochondrial dysfunction, axon abnormalities, and oxidative stress, have been implicated in the motor neuron degeneration of ALS [7-10]. However, most SOD1 transgenic mouse models overexpress mutant SOD1, and their relatively short life spans may preclude development of the full range of ALS neuropathology seen in patient brains. For example, SOD1 (G93A) mice that express 18 copies of the transgene show fourfold higher SOD1 enzymatic activity than their non-transgenic littermates [3]. These transgenic SOD1 mouse models carry a greatly elevated protein load compared with human ALS tissues, and these animals exhibit some phenotypic features, such as intracytoplasmic vacuoles derived from the mitochondria and the endoplasmic reticulum, which are rarely found in human ALS tissues [11-13]. In terms of the mechanisms of neuronal death in ALS, most evidence from mouse models suggests that apoptosis may play a crucial role in the death of motor neurons [14-16]; however, conclusive evidence of apoptosis has been difficult to detect in humans [17-20]. In addition, intranuclear inclusions were seen in the brains of ALS patient with SOD1 mutations [21-23], which have nevertheless not been found in transgenic mutant SOD1 mouse models. These pathological differences could be due to species differences in anatomy, genomics, and physiology between small animals and humans and may also explain why some therapies uncovered using SOD1 mouse models have been hard to translate to the treatment of ALS patients, thus it is important to explore whether large animal models can mimic the more pathological changes seen in ALS patients.

The pig has several unique features that make it a promising alternative animal model $[24,25]$, with a rich history of use in human biomedical research including neuroscience, because of its considerable anatomical and genetic similarities to humans. Moreover, the recent development of somatic cell nuclear transfer (SCNT) technology has made it possible to efficiently generate genetically modified large animals. The successful cloning and production of genetically modified pigs by nuclear transfer of transgenic or gene-targeted somatic cells have been reported [26], including transgenic Huntington's disease pigs generated in our previous studies [27]. In the current study, we used the SCNT method to generate transgenic pigs that express autosomal-dominant fALS mutants of SOD1 (G93A). The transgenic pigs develop age-dependent movement disorders, which recapitulate the features of an early disease symptom seen in human
ALS, as well as neuropathology. Moreover, transgenic mutant SOD1 pigs show intranuclear inclusions and an association of SOD1 with the nuclear protein, PCBP1, which are not seen in the mouse brains. Our findings suggest that species-specific targets of misfolded proteins may account for the ALS neuropathology that is unique to large mammals and humans, underscoring the importance of transgenic large animals in unraveling disease pathogenesis and identifying therapeutic targets.

\section{Results}

Generation of human SOD1 (hSOD1) (G93A) transgenic pigs

SOD1 is a protein widely expressed in mammal tissues. We used the cytomegalovirus (CMV) promoter to ubiquitously express transgenic hSOD1 in pig and introduced the G93A mutation, which has been used principally to create genetic animal models of ALS, into the human SOD1 transgene using a site mutagenesis technique. SV40 early mRNA polyadenylation signal was used as the transcription termination for transgene, and the neomycin resistance gene was included as the selection marker for screening stably transfected cell lines (Supplementary information, Figure S1A). Primary pig fetal fibroblasts isolated from 35-day-old Tibetan miniature pig fetus were transfected with linearized hSOD1 transgene and selected with $800 \mu \mathrm{g} / \mathrm{ml} \mathrm{G} 418$ for about 2 weeks. We identified the transgenic cell lines that expressed the SOD1 transgene as donors for SCNT (Supplementary information, Figure S1B) and transferred a total of 1635 SCNT embryos into 11 surrogates that exhibited natural estrus. Eight surrogates developed to term and gave birth to 37 female Tibetan miniature piglets after 120-130 days of gestation. Of these, 15 piglets including wild-type ones died at birth or 1 week after birth and appeared to have small and weak bodies. As a result, 22 healthy piglets survived until ablactation (Figure $1 \mathrm{~A}$ and Supplementary information, Figure S1C). Most live piglets appeared normal at birth, and there was no obvious difference in appearance between the cloned and naturally bred piglets.

PCR analysis of DNA samples obtained from the ear tissues of each piglet showed that the live piglets, except TG-4, were positive for the SOD1 transgene. Real-time PCR of transgene copy numbers revealed that transgenic pigs carried various numbers $(1-20)$ of the transgene (Supplementary information, Table S1). Using immunohistochemistry staining with an antibody specific to hSOD1, we could confirm the expression of transgenic hSOD1 in the lumbar spinal cords of a stillborn transgenic pig, but not in a wild-type one (Figure 
A

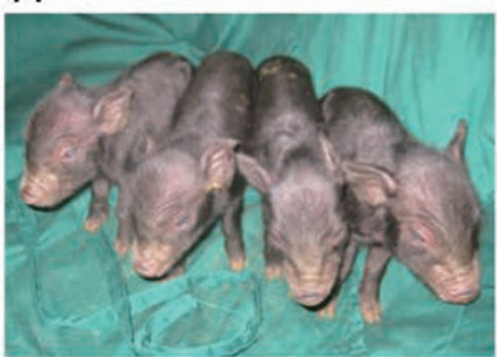

B

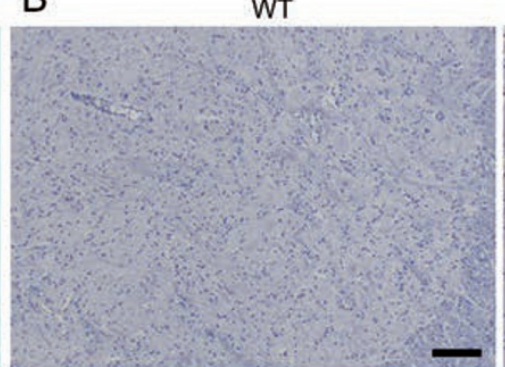

TG (504-1)

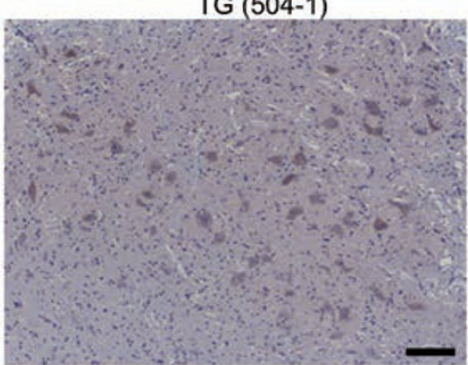

C
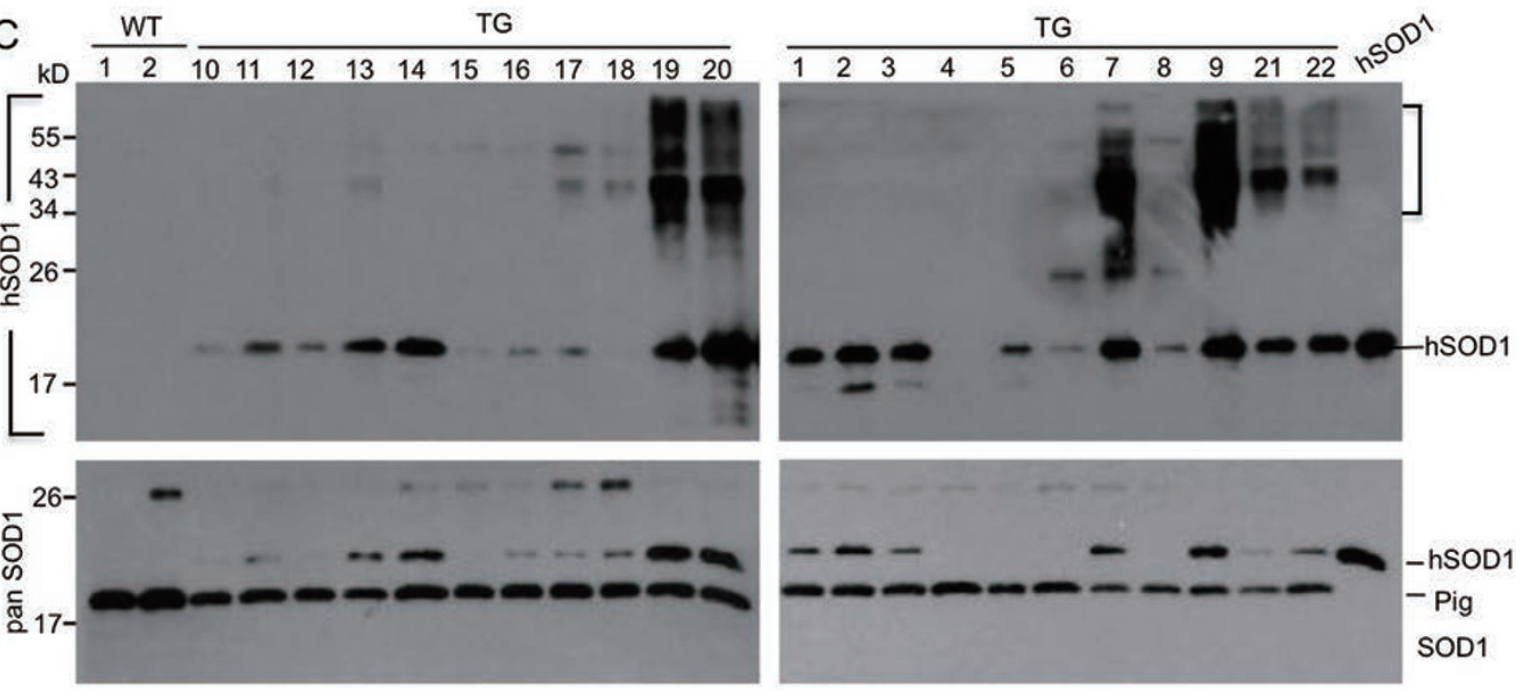

D

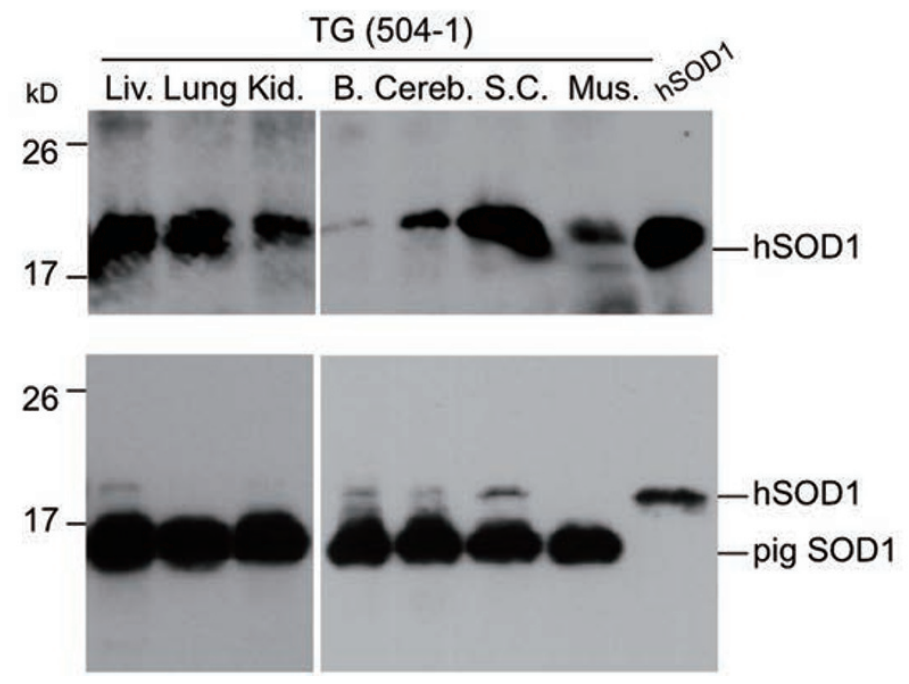

Figure 1 Generation of hSOD1 (G93A) transgenic pigs. (A) A photograph of four newborn transgenic pigs (TG-16, 17, 18, and 19) was taken on the first day after birth. (B) Immunohistochemical staining of lumbar spinal cord of a stillborn transgenic pig (\#504-1) with anti-human SOD1 antibody showing the expression of hSOD1 protein in the lumbar spinal cord of transgenic pig. Scale bars, $200 \mu \mathrm{m}$. (C) Western blots of ear biopsy lysates from WT and 22 transgenic pigs using antibodies that detect either hSOD1 only (upper panel) or both endogenous pig SOD1 and transgenic hSOD1 (lower panel, pan SOD1). Endogenous pig SOD1 immunoreactivity shows equal protein loading in each lane. hSOD1-transfected HeLa cell lysates served as the positive control. Note that hSOD1 in transgenic pig tissues shows a dimerized form ( 40 kDa) and oligomeric forms with high molecular weight. (D) Western blot analysis showing hSOD1 ubiquitously expressed in the brain regions and peripheral tissues. In a stillborn transgenic pig (TG \#504-1), transgenic hSOD1 is expressed at levels lower than endogenous pig SOD1. Liv., liver; Kid,, kidney; B, whole brain; Cereb., cerebellum; S.C., spinal cord; Mus., muscle; hSOD1, transfected hSOD1. 
1B). Western blot analysis of the ear tissue lysates of live pigs confirmed the expression of hSOD1 in 21 transgenic piglets (Figure 1C). The blots were probed with an antibody specific to transgenic hSOD1 and a pan-antibody that recognizes both endogenous pig SOD1 and transgenic hSOD1. With the antibody to hSOD1, we found that several pigs' tissues contained a dimerized band (44 $\mathrm{kDa}$ ), which is about double the monomeric hSOD1 (22 $\mathrm{kDa}$ ), and a smear that represents high-molecular-weight forms of oligomerized SOD1 (Figure 1C). Western blot analysis also revealed that transgenic SOD1 is expressed in various peripheral tissues and brain regions, consistent with the ubiquitous expression of mutant SOD1 in ALS patients (Figure 1D). Importantly, western blotting with the pan-antibody also revealed that the levels of transgenic hSOD1 are lower than or equivalent to that of endogenous pig SOD1 (Figure 1C and 1D), indicating that the transgene is not overexpressed in our transgenic pig models.

\section{Limb movement defect in transgenic SOD1 pigs}

Perhaps because transgenic hSOD1 is not overexpressed, transgenic SOD1 pigs grew normally and did not show obvious body weight differences from wild-type pigs (Supplementary information, Table S2). At present, the oldest transgenic SOD1 pig has been living for about 2 years. We saw no early death due to transgenic gene expression in these transgenic pigs.

Since ALS is characterized by progressive loss of upper and lower motor neurons, which results in limb movement impairment and paralysis, we examined the hind limb capacity of SOD1 transgenic pigs for movement by treadmill test. At the age of 3 months, some transgenic pigs showed a running defect on a treadmill. The major manifestation was hind limb weakness, which prevented the pig from running on the treadmill, and this weakness became more severe with age. As shown in Figure 2A and Supplementary information, Movies S1S4, the hSOD1 transgenic pigs leaned against the back wall of the treadmill on the rear end or sit on their hind legs, indicating an inability to run. With a gradual acceleration of running speed (from $1.0 \mathrm{~km} / \mathrm{h}$ to $6.0 \mathrm{~km} / \mathrm{h}$ over $60 \mathrm{~s}$ ) to measure the maximal speed at which a pig was able to run, we could quantify the pig's limb movement capacity. By comparing different wild-type (WT) and transgenic pigs, we found that most transgenic pigs, except TG-1 and TG-10, had a significant reduction in their maximal speeds (Figure 2B). We also used a constant speed $(3.0 \mathrm{~km} / \mathrm{h})$ to examine the length of time during which a pig could not run (or time of running deficit) over a 60 -s test period. This test also showed significantly increased times of running defects for most transgenic pigs (Figure 2C and Supplementary information, Movies S1-S2). Also, transgenic pigs (TG-6 and TG-9) show age-dependent worsening in treadmill running, while other hSOD1 transgenic pigs had different degrees of running impairment (Figure 2D). These results indicate that the treadmill tests were reliable and repeatable for identifying the severity of motor deficits in hSOD1 transgenic pigs.

We were able to collect ear tissues of four different transgenic pigs (TG-1, TG-6, TG-9, and TG-16) while monitoring their treadmill performance at different ages (newborn, 3, 7, and 19 months). Western blot analysis showed that oligomeric SOD1, which reflects the accumulation of misfolded proteins, is most abundant in the TG-16 pig from 3 months and less abundant in TG-9 and TG-6 pigs (Figure 2E and 2F). The TG-1 pig expresses the least amount of transgenic SOD1 (Figure 2E and $2 \mathrm{~F})$. Consistent with their different levels of transgenic hSOD1, the TG-16 pig showed a more severe running defect than TG-9 and TG-6 pigs, while the TG-1 pig displayed the best running ability (Figure 2D). The transgenic pigs with the higher level of misfolded proteins also exhibited the earliest running disability. However, the TG-6 pig with high expression levels of hSOD1 showed a significant running defect from 19 months of age compared with its performance at younger ages (3-7 months) (Figure 2D). All these findings indicate that high levels or accumulation of mutant SOD1 leads to neurological symptoms in transgenic SOD1 pigs, a phenotype that is consistent with disease progression in humans.

Breeding transgenic SOD1 pigs yielded two F1 lines of pigs from TG-7 and TG-11 founders. PCR genotyping verified the germline transmission of the transgenes in some F1 pigs (Figure 3A). These F1 pigs are currently at 9 months of age. Western blot analysis of ear tissues using anti-SOD1 also showed the expression of transgenic SOD1 in these F1 pigs that are positive for their genotyping (Figure 3B). However, these F1 pigs appeared to express either low or high levels of mutant SOD1, suggesting that they carry different copy numbers of the transgene due to differential segregation of chromosomes, which harbor different copies of the transgene, from the founder to the offspring. Indeed, real-time PCR suggested that $\mathrm{F} 1$ pigs (7-2, 7-3, 7-4, 7-6) expressing the low level of mutant SOD1 carry one copy while those (7-1, 7-5, 7-7) expressing the high level carry about eight copies of the transgene (Supplementary information, Table 1). We then used the treadmill to test limb movement of F1 pigs that were trained at 3 months of age (3 months old) and examined once every month for four times. Some F1 pigs (7-2 and 7-6) were not included in the statistical analysis because of their insufficient train- 
A

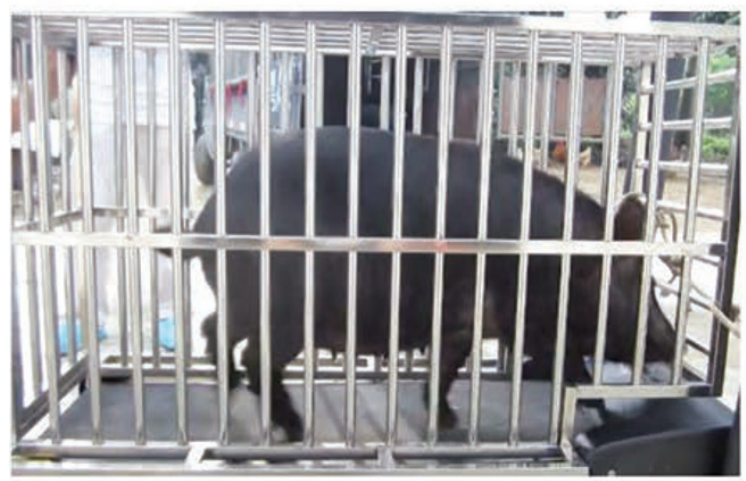

TG $16 \mathrm{~m}$

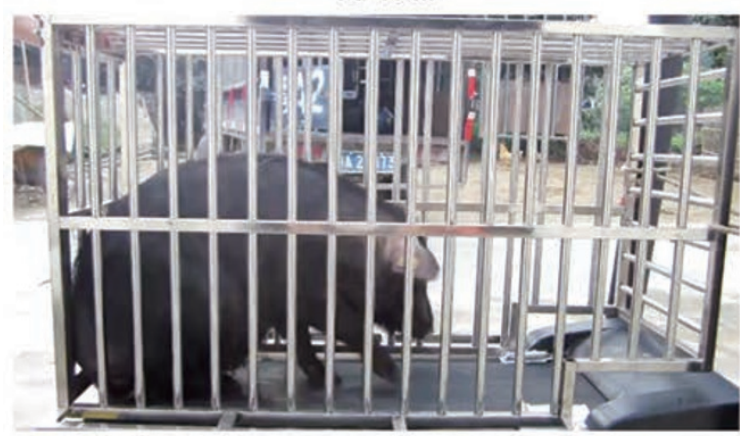

E

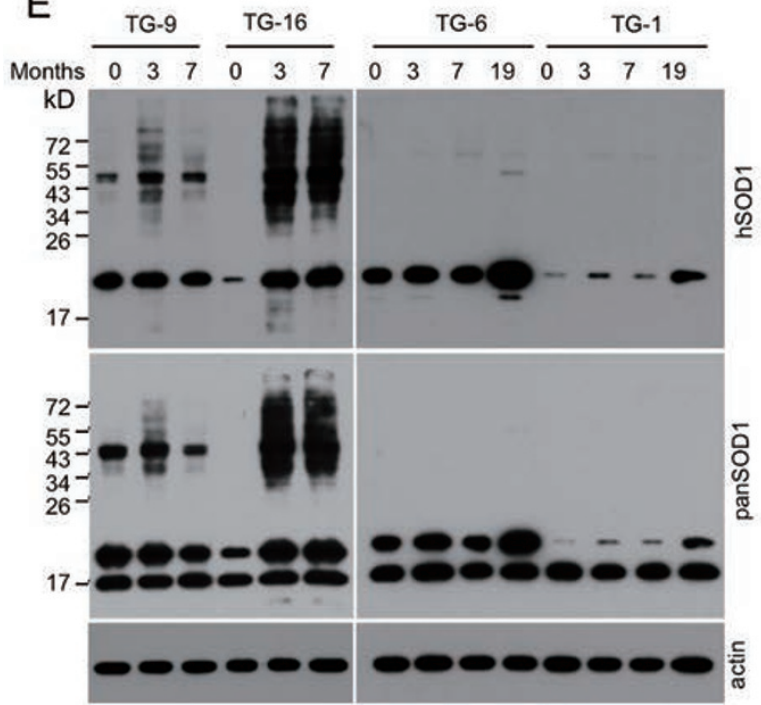

B

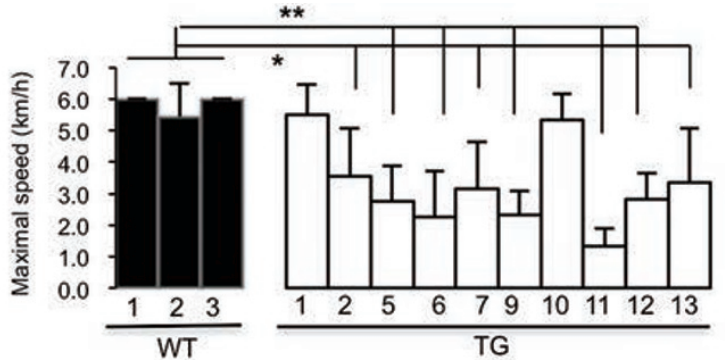

C

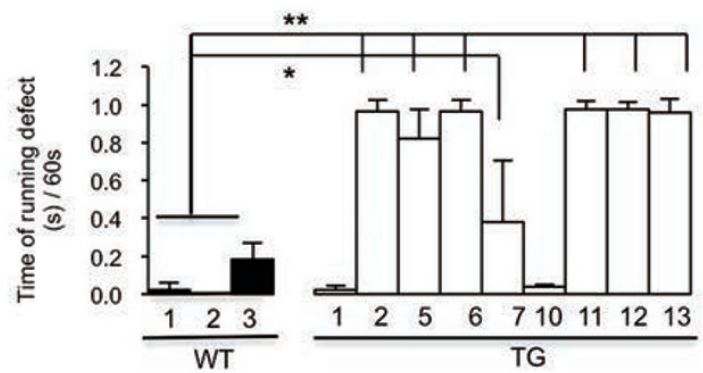

D

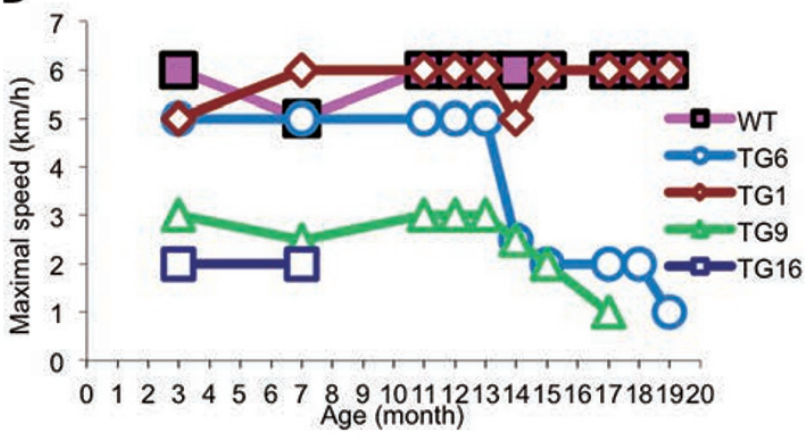

F

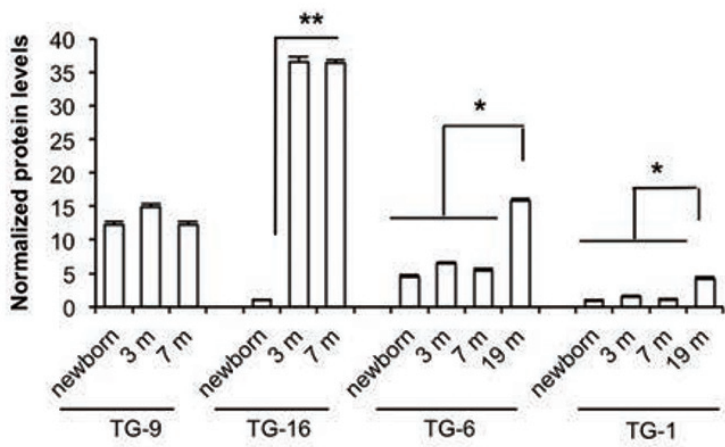

Figure 2 Limb movement defects in hSOD1 transgenic pigs. (A) Representative photos of wild-type (WT) and hSOD1 transgenic (TG-11) pigs at 16 months of age on treadmill (also see Supplementary information, Movies S1-S4). (B, C) The accelerating (B) and constant (C) speed treadmill running tests revealed obvious motor defects of hSOD1 transgenic pigs in the maximum speed and duration in treadmill running tests. Accelerating speed treadmill running data are the means of nine tests from 11 to 19 months for each living pig. Constant speed treadmill running data are the means of three tests from 17 to 19 months for each living pig. Values are reported as mean \pm SEM. ${ }^{*} P<0.01,{ }^{* *} P<0.001$, by one-way ANOVA with Bonferroni's post hoc test. (D) Maximal speed of transgenic pigs in treadmill running test also demonstrates progressive motor defects with age (months) for hSOD1 transgenic pigs. (E) Representative SOD1 immunoblots of hSOD1 levels in ear biopsy homogenates of hSOD1 transgenic pigs showing the age-dependent accumulation of misfolded hSOD1 and different levels of transgenic hSOD1 in pigs. (F) hSOD1 levels (oligomeric and monomeric) in western blots are normalized by $\beta$-actin levels and shown as ratio to $\beta$-actin (means \pm SEM, ${ }^{*} P<0.01,{ }^{* *} P<0.001$ by Student's paired $t$-test), which indicate that transgenic hSOD1 of TG-16 pig increases and accumulates rapidly over 3 months. 
A
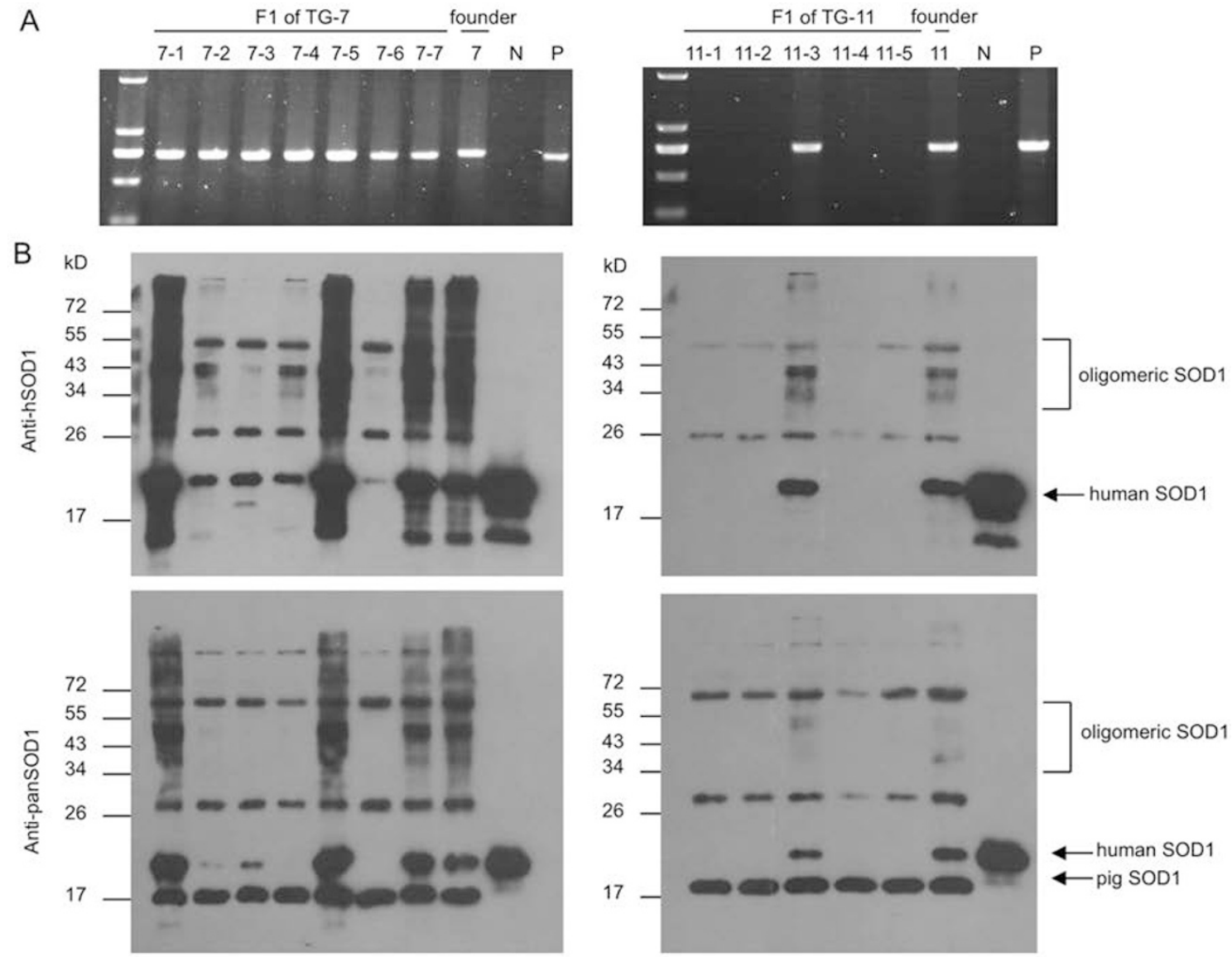

C

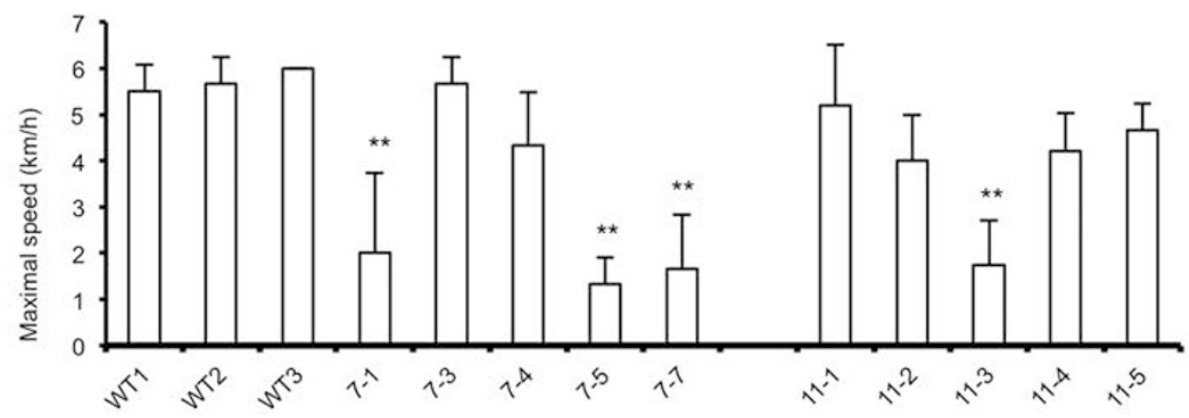

Figure 3 Germline transmission of mutant SOD1 and motor deficit in transgenic F1 pigs. (A) Genotyping analysis results of F1 pigs born by TG-7 and TG-11 transgenic pigs. P is the PCR result from hSOD1 plasmid, which served as a positive control. $\mathrm{N}$ is negative control. (B) Western blot analysis of the expression of mutant SOD1 in transgenic F1 pig ear tissues. Antibodies to human SOD1 (upper panel) and both pig and human SOD1 (pan-SOD1, lower panel) were used to probe western blots. (C) Treadmill tests showing the impairment of hind limb movement of F1 transgenic hSOD pigs. F1 transgenic pigs with higher transgene expression (7-1, 7-7, 11-3 are male; 7-5 is female) showed more severe motor defects than those expressing lower levels of mutant SOD1. Data (mean \pm SEM, $n=4$ ) of WT and F1 of TG-7 or TG-11 are the average value from pigs at the age of 5-8 months. ${ }^{* *} P<0.001$ vs WT by one-way ANOVA with Bonferroni's post hoc test.

ing and tests or early death. Although the expression levels of transgenic SOD1 in ear tissues do not necessarily correlate with the expression levels of transgenic SOD1 in the brain and spinal cord tissues, more F1 pigs from the TG-7 founder $(7-1,7-5,7-7)$ showed higher levels of transgenic mutant SOD1 than those from the TG-11 founder (11-1, 11-2). We examined the motor function of these F1 pigs using the treadmill assay. When running in an accelerating speed mode on treadmill, transgenic pigs showed obviously decreased maximal speed com- 
pared with age-matched WT. Importantly, F1 pigs (7-1, 7-5, 7-7, 11-3) with higher transgene expression levels showed more severe motor defects than lower expressing ones such as 7-3, 7-4, 11-1 and 11-2 transgenic pigs (Figure $3 \mathrm{C}$ ). Thus, we have established transgenic SOD1 pigs in which transgenic SOD1 gene is germline transmissible and causes motor deficit.

\section{Limb muscle atrophy in transgenic SOD1 pigs}

Since motor neuron degeneration can lead to denervation of muscle and muscle atrophy in ALS, we next isolated muscle sample from the gastrocnemius and soleus muscle from TG and WT pigs for examination. Hematoxylin and eosin staining revealed differences in gastrocnemius muscle mass between wild-type and SOD1 transgenic pigs. The TG-16 pig at 8 months of age and TG-11 pig at 22 months of age showed a reduction in cross-sectional area of the muscle fibers compared with age-matched WT pigs (Figure 4A and Supplementary information, Figure S2). Skeletal muscle molecular biomarkers, NCAM and Nogo-A, are known to be increased in the denervated muscle in ALS [28-30]. These marker proteins were also increased in the muscle sections from our TG-16 pig compared with WT pig (Figure 4A). Morphometric analysis showed that Nogo-A levels correlated with the mean area of fast-twitch type II fibers, and many of them are small and present an angulated morphology (Figure 4B). These data were identical to an earlier observation that fast-twitch muscle fibers were preferentially affected by ALS-induced denervation [31].

We hypothesized that the muscle atrophy might be due to abnormal myofibrillar protein degradation induced by the ubiquitin-proteasome pathway dysfunction. Western blotting of the hind limb gastrocnemius muscle showed enhanced expression levels of Nogo-A in fibers from 8-month-old TG-16 transgenic pig, but not agematched WT pigs (Figure 4C). This assay also revealed a pronounced decrease in actin (including $\beta$-actin and skeletal muscle actin) and the apparent degradation of myosin heavy chain (MyHC) into low-molecular-weight fragments in TG-16 transgenic pigs. Meanwhile, two muscle-specific E3 ubiquitin ligases, atrogin-1/MAFbx and MuRF1, were markedly upregulated in TG-16 atrophic muscle (Figure 4C). These findings reflect altered function of the ubiquitin-proteasome system, which in turn induces abnormal protein degradation in the skeletal muscle of transgenic pigs.

To measure motor unit stimulation, we performed quantitative electromyography (EMG), which detects action potential parameters, in the bilateral tibialis anterior muscle and the right sternocleidomastoid muscle of two transgenic pigs (TG-5 and TG-9) at 8 months of age and two age-matched wild-type pigs. In the two hSOD1 transgenic pigs, we detected fibrillation potentials and positive sharp waves (Figure 4D), which are the most significant EMG features in the very early stage of ALS [32]. Statistical analysis clearly showed increases in potential amplitude (Amp), duration (Dur), and polyphases of MUPs in hSOD1 transgenic pigs (Figure 4E), which reflects abnormal motor neuron inneveration of muscle.

\section{Neurodegenerative pathology in hSOD1 transgenic pigs}

We sacrificed several pigs to investigate the neuropathology caused by mutant hSOD1. The transgenic pigs demonstrated accumulation of hSOD1 in motor neurons in the ventral horn of lumbar spinal cords (Supplementary information, Figure S3A).

Using anti-ChAT immunohistochemistry, we found no significant difference in the numbers of ChAT-positive motor neurons between 4-month-old hSOD1 (G93A) transgenic pigs and age-matched wild-type controls. However, there was an obvious decrease in ChATpositive large motor neurons in the ventral horn of the lumbar spinal cords in 8-month-old hSOD1 transgenic pig (Supplementary information, Figure S3A). The number of ChAT- and NeuN-positive motor neurons was significantly decreased in 8-month-old TG-16 transgenic pig by about $50 \%$ of the WT number (Figure 5B and Supplementary information, Figure S3B). Also, we found obviously fewer neurons in the intermediate zone of lumbar spinal cord in transgenic pigs (Figure 5A and 5B). In 8-month-old TG-16 transgenic pigs, anti-NeuN immunohistochemistry showed that numbers of spinal interneurons in laminae (L) VI and VII were reduced. Quantification of the interneurons (diameter $<25 \mu \mathrm{m}$ ) in laminae VI and VII showed a $30 \%$ reduction in TG-16 transgenic pig (Figure 5B). NeuN-labeled motor neurons, which were readily identifiable as large size neurons $(>$ $25 \mu \mathrm{m}$ ) located in lateral laminae IX, were significantly reduced in TG-16 pig (Figure 5B). Immunostaining the specific interneuron markers parvalbumin (PV), calbindin (CB), and calretinin (CR) could detect the loss of PV- and CR-labeled interneurons in the lumbar spinal cords of transgenic pigs (Supplementary information, Figure S3A and S3B). There was also a marked increase in Iba-1 and GFAP staining, which reflects microgliosis and astrocytosis, respectively, in the lumbar spinal cord of 8-month-old TG-16 transgenic pig (Figure 5C). This finding suggests that a glial proliferation occurs in response to neuron degeneration in these regions. Furthermore, electron microscopy provided evidence for demyelinating axons and degenerated neuronal cells in the ventral horn of lumbar spinal cord, which were not seen in the age-matched wild-type pig spinal cord (Figure 


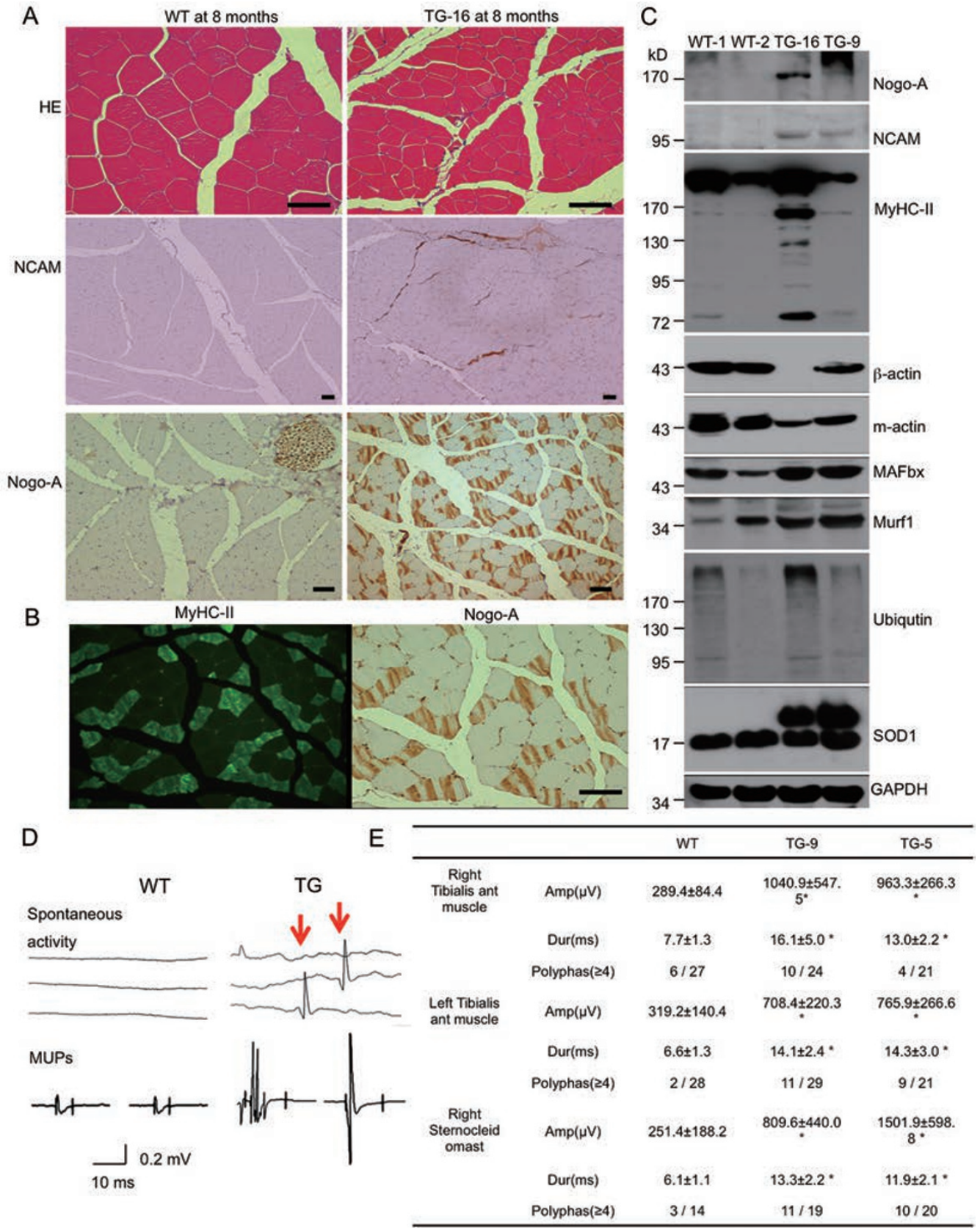

Figure 4 Skeletal muscle atrophy in hSOD1 transgenic pigs. (A) Hematoxylin and eosin (HE) stain of muscle from 8-monthold TG-16 transgenic and WT pigs showing a significant reduction in the cross-sectional area of gastrocnemius muscle fibers in TG-16 muscle compared with WT muscle. Immunohistochemical studies also show enhanced NCAM and Nogo-A levels in the muscle of TG-16 pig, but not in WT pig. Scale bars, $100 \mu \mathrm{m}$. (B) Double immunostaining showing that Nogo-A is localized in the fast-twitch (type II) fibers that also contain MyHC-II staining signals. Scale bars, $100 \mu \mathrm{m}$. (C) Western blot analysis of expression levels of Nogo-A, NCAM, Myosin, $\beta$-actin, skeletal muscle actin (m-actin), Murf1, MAFbx, ubiquitin, and SOD1 in the gastrocnemius muscle homogenates from two wild-type (WT-1 and WT-2), TG-16, and TG-9 transgenic pigs. Note that TG-16 pig shows obvious muscle myofibrillar protein degradation, and upregulation of ubiquitin as well as muscle-specific E3 ubiquitin ligases atrogin-1/MAFbx and MuRF1. (D) Electromyography of tibialis anterior muscles or sternocleidomastoid muscles revealing frequent fibrillation potentials (arrows) in transgenic (TG) pigs, but not in WT controls. MUP parameters show increased potential duration, amplitude, and polyphasia. (E) Quantitative analysis of MUP parameter data in transgenic pigs and WT pigs. Two transgenic pigs show increases in duration, amplitude, and polyphasia of MUPs compared with WT pig. At least 20 MUPs were recorded and analyzed for each pig. Data are presented as means \pm SEM. ${ }^{*} P<0.001$ vs WT group by one-way ANOVA with Bonferroni's post hoc test. 
A

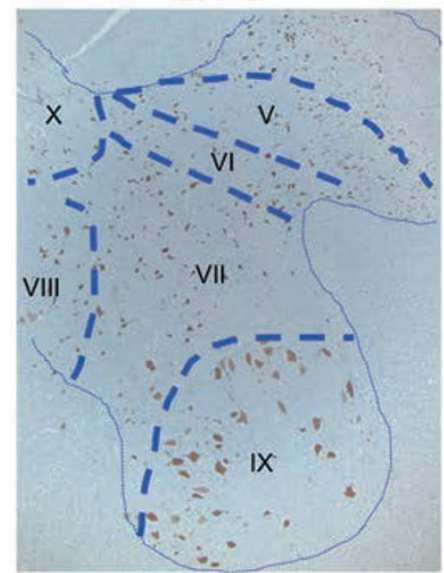

C
TG-16 8m

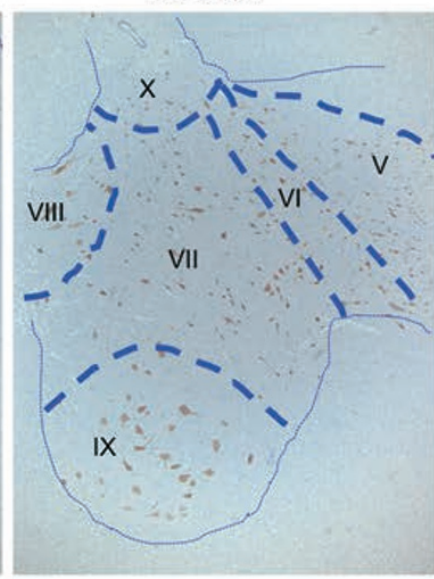

Control
B

ChAT motor neurons

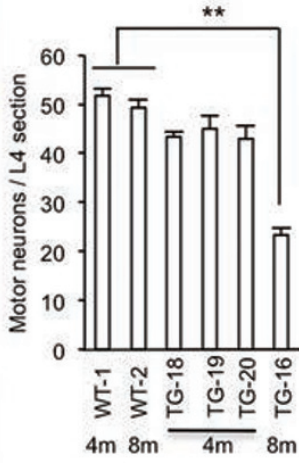

TG
NeuN neurons

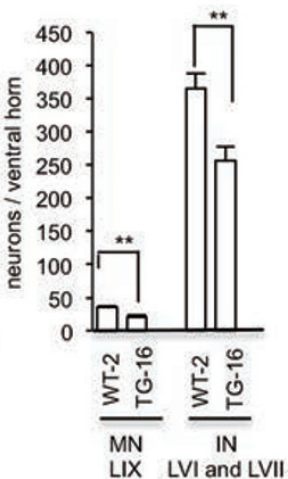

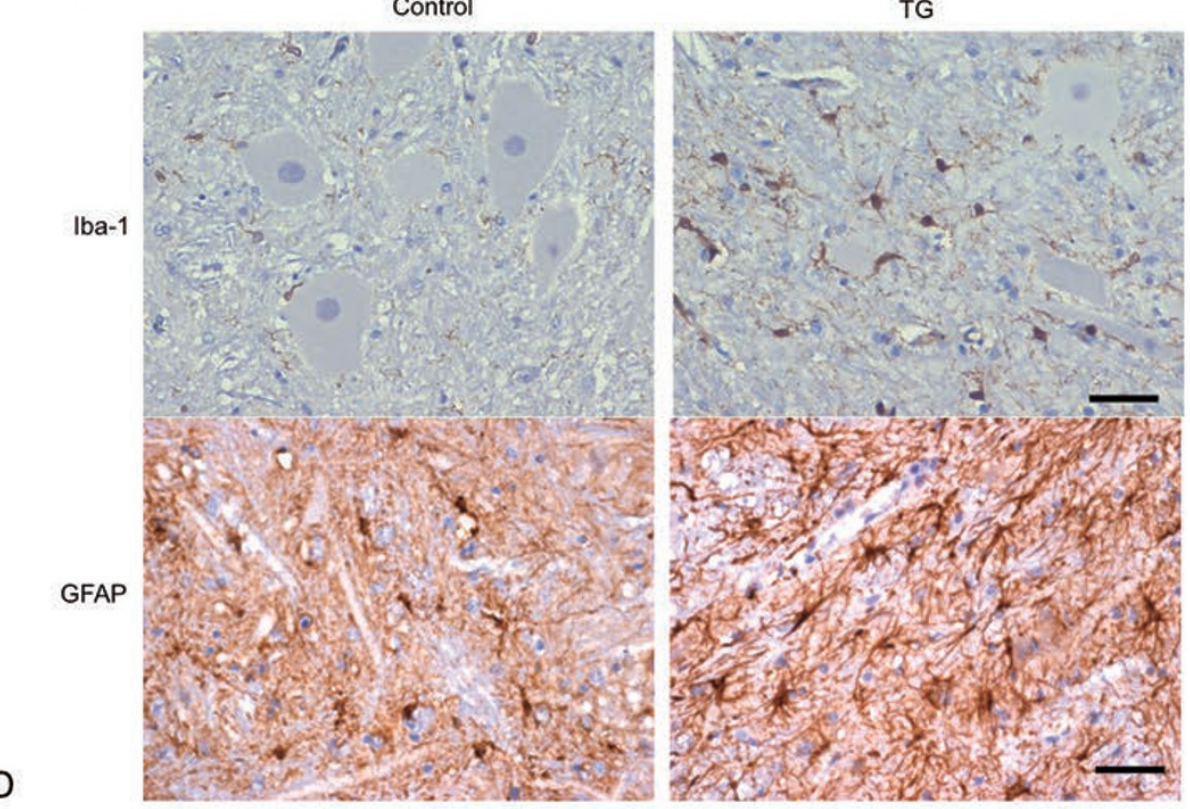

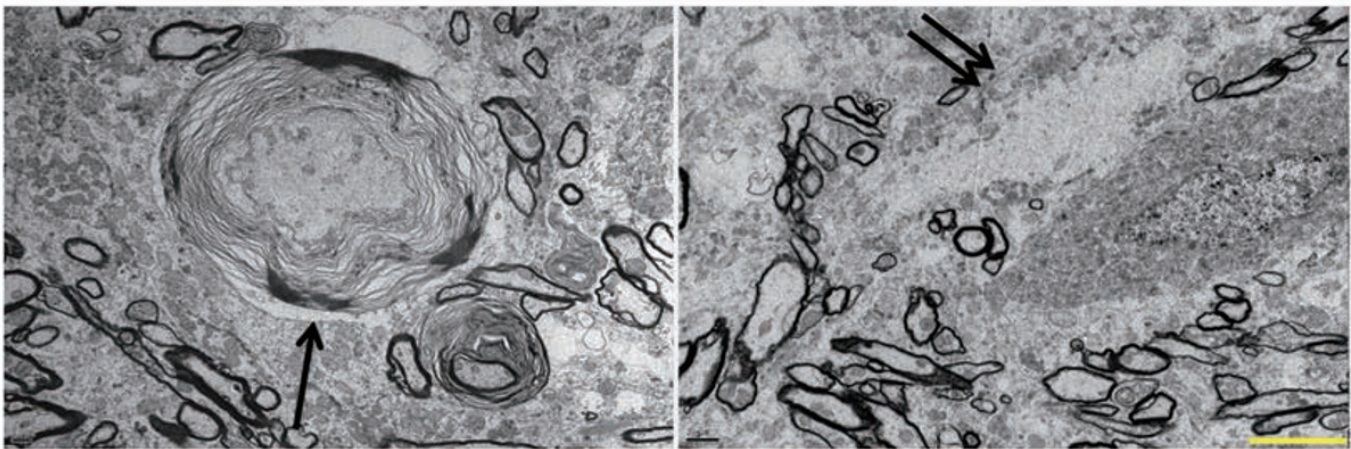

Figure 5 Neuropathology in hSOD1 transgenic pigs. (A) Reduced NeuN immunoreactivity is present in the ventral horn of TG-16 pig at the age of 8 months compared with the age-matched WT pig. Blue broken arrow lines define different regions in the ventral horn (LIX) and intermediate zone (LVI and LVII) of the spinal cord. (B) Quantitative assessment of the number of ChAT- and NeuN-positive cells. More data are also provided in Supplementary information, Figure S3. Data are the means \pm SEM of three L4 slices; ${ }^{* *} P<0.001$, as assessed by a Student's $t$-test. (C) Enhanced Iba- 1 and GFAP immunoreactivity indicating activated microglia and reactive astrogliosis, respectively, in the spinal cord of TG-16, but not WT, pig. (D) Electron microcopy showing demyelinating axon (arrow in left panel) and degenerated neuronal cell (double arrows in right panel) in the spinal cord of TG-16 transgenic pig. Scale bars: $50 \mu \mathrm{m}$ in $\mathbf{C}$ and $5 \mu \mathrm{m}$ in $\mathbf{D}$. 
5D and Supplementary information, Figure S4A). Using an antibody to activated caspase-3 and TUNEL staining, we did not find clear evidence for the apoptotic neurons in hSOD1 transgenic pig brain and spinal cord (Supplementary information, Figure S4B). Although we did not identify apoptotic events in hSOD1 transgenic pig brains, other potential pathological changes via non-apoptotic mechanisms, such as ER stress, remain to be investigated.

Formation of neuronal intranuclear inclusions (NIIs) in transgenic pigs

Immunohistochemistry study showed prominent expression of mutant hSOD1 in the cytoplasm. However, we did not detect inclusions in the cytoplasm of these neurons. Instead, some motor neurons contained nuclear inclusions (arrows in Figure 6A). Double immunofluorescent staining indicated that these nuclear SOD1 inclusions were also labeled by anti-ubiquitin (Figure 6B). These inclusions were not labeled by antibodies to $\alpha$-synuclein, ataxin-3, neurofilaments, polyglutamine repeat, or TDP-43 (data not shown). Immunofluorescent staining verified that these nuclear inclusions were not present in wild-type pig spinal cord (Figure 6C). In TG-9 pig at 17 months of age, hSOD1-positive intranuclear inclusions were seen in the neural cells of the spinal cord. Costaining with anti-ubiquitin antibody demonstrated that the inclusion bodies were exclusively localized in the nucleus of neuronal cells, and the inclusions were found in larger motor neurons in the ventral horn and smaller bipolar or multipolar neurons, which may be interneurons, in the intermediate zone and dorsal horns (Figure 6D). Moreover, these protein inclusions could be confirmed by western blot analysis using SOD1 and ubiquitin antibodies, which show oligomeric forms of hSOD1 and increased ubiquitination in TG-16 pig brain tissues (Figure 6F and 6G). When comparing with the endogenous pig SOD1 on the same western blots, it is noticeable that the expression levels of mutant hSOD1 in the spinal cord and brain in TG-16 pig appear to be higher than that in ear tissues seen in Figure 1C.

Double immunofluorescent staining also showed that the larger neurons containing nuclear ubiquitinated inclusions were ChAT-positive (Figure 7A), and the smaller neurons are CR- and PV-positive (Figure 7B and 7C) interneurons. Ubiquitin-positive nuclear inclusions were also abundant in neurons of cortical layers (Figure 6E), but were not found in peripheral tissues, including liver, heart, muscle, and kidney (data not shown). By counting the numbers of nuclear inclusion-containing neurons in the ventral horn, dorsal horn, and intermediate zone of the lumbar spinal cord, we also provide evidence for the presence of these nuclear inclusions in the large motor neurons in the ventral horn, and interneurons in the dorsal horn and intermediate zone (Figure 7D and 7E), which is consistent with the accumulation of mutant hSOD1 in these neurons in hSOD1 transgenic pigs.

\section{hSOD1 interacts with PCBP1 in pig brains}

The nuclear accumulation of hSOD1 and its formation of nuclear inclusions in transgenic pig brains are similar to the presence of ubiquitinated nuclear inclusions of SOD1 in the brain and spinal cord tissue of ALS patients $[33,34]$. Such nuclear SOD1 inclusions, however, have never been found in transgenic SOD1 mouse brains. We hypothesized that species-specific targets may account for the nuclear SOD1 inclusions in larger mammals. To test this hypothesis, we compared SOD1-interacting proteins between mouse and pig brains using GST pull-down assay. GST-hSOD1 was used to pull down proteins from mouse or pig cortex lysates. Silver staining revealed an additional band (arrow in Figure 8A), which was specifically present in the pull-down from the pig cortex tissue. This band was isolated for mass spectrometry and found to contain Poly(rC)-binding protein 1 (PCBP1), a nuclear RNA-binding protein [35-37]. We then performed GST pull-down with an antibody to PCBP1, which confirmed that pig PCBP1, but not mouse PCBP1, bound to hSOD1 (Figure 8B). To further verify this differential binding, we used anti-SOD1 to immunoprecipitate mouse or pig brain cortex lysates and also found that PCBP1 in pig brain was markedly coprecipitated with SOD1 compared with mouse PCBP1 (Figure 8C). All these results indicate that the interaction of hSOD1 with PCBP1 selectively occurs in pig brain, but not in mouse brain.

Given the selective interaction of PCBP1 with hSOD1 in pig brain, we wanted to examine whether the nuclear localization of PCBP1 is altered in hSOD1 transgenic pig brain. We performed PCBP1 immunocytochemical studies and found that PCBP1 is normally diffuse in the cytoplasm and nuclei of motor neurons in the spinal cord. However, in hSOD1 transgenic pig motor neurons, we saw more neurons containing nuclear PCBP1 inclusions (Figure 8D). In immunofluorescent double staining, we also verified that hSOD1 nuclear inclusions in transgenic pig brain were labeled by anti-PCBP1 (Figure $8 \mathrm{E})$. Quantitative analysis of the numbers of motor neurons containing PCBP1 nuclear inclusions also show that a significantly greater number of neurons display PCBP1 inclusions in hSOD1 transgenic pig spinal cord (Figure $8 \mathrm{~F})$. Taken together, these results support the increase in the number of nuclear inclusions and the interaction of hSOD1 with PCBP1 in hSOD1 transgenic pig brains. 

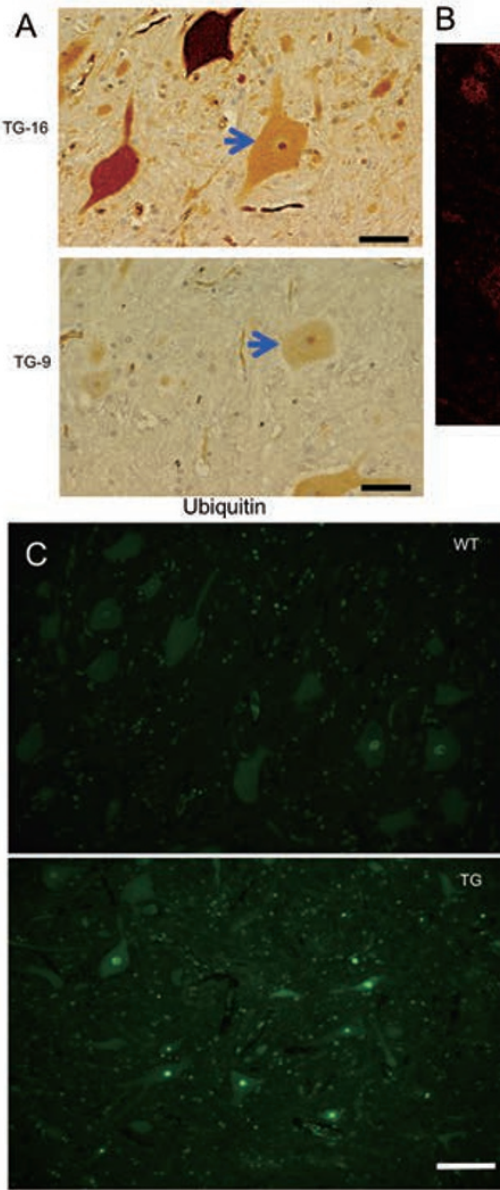

F

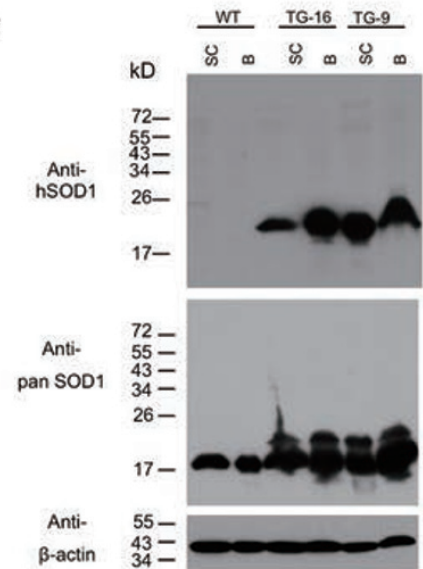

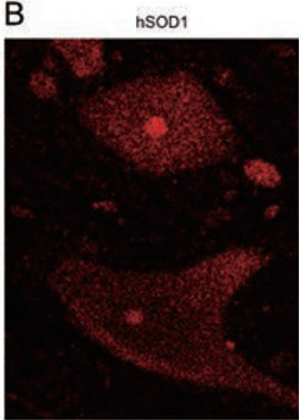
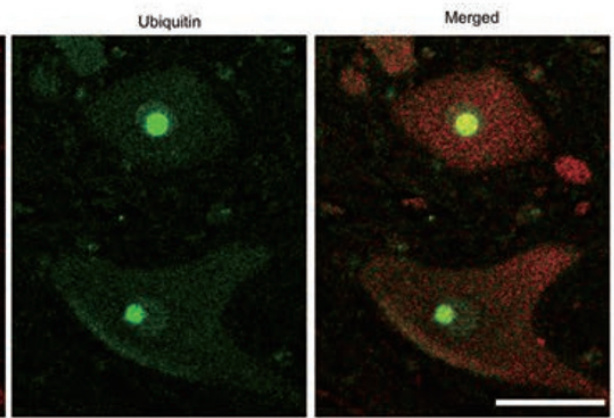

Ubiquitin
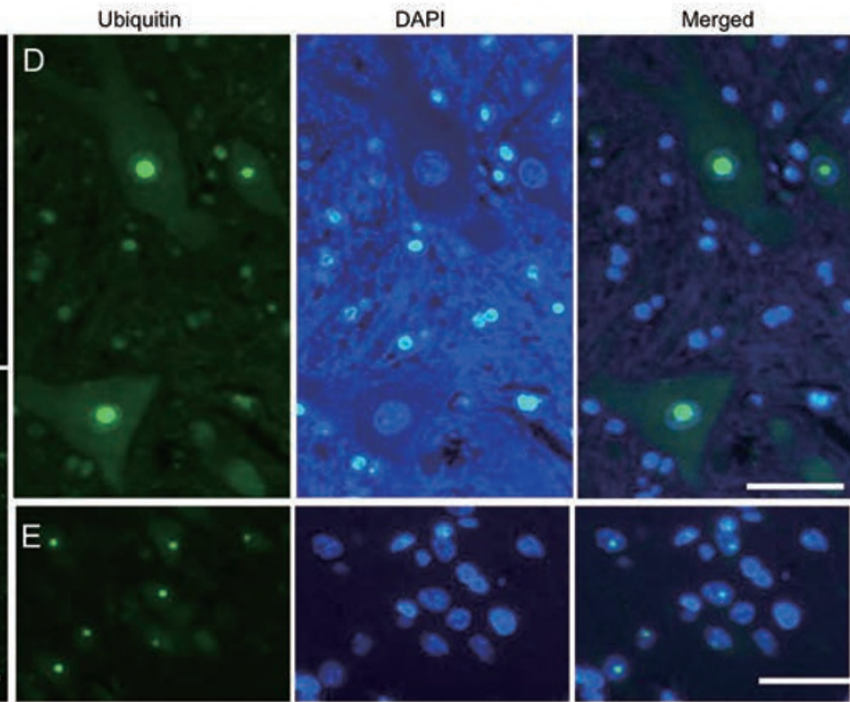

Ant-ubiquitin
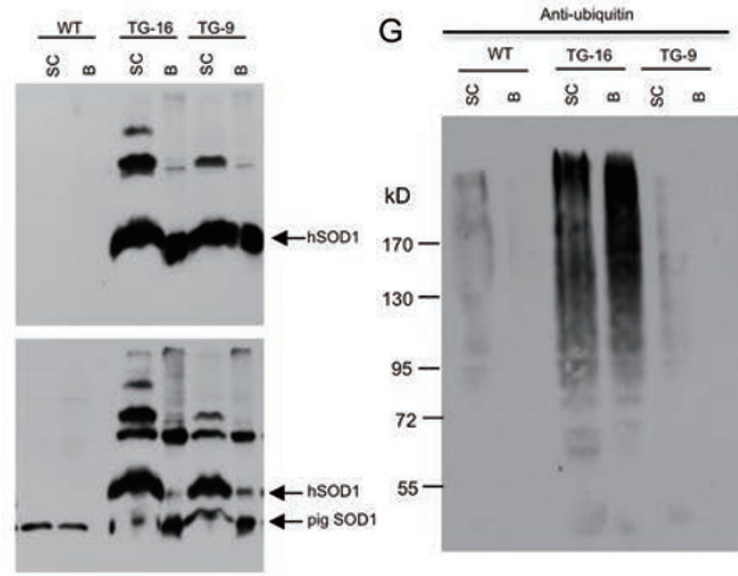

Figure 6 Nuclear accumulation and inclusions of hSOD1 in transgenic pig brain and spinal cord. (A) Immunocytochemical studies revealing that, in addition to its cytoplasmic distribution, hSOD1 forms intranuclear inclusions (arrows) in the spinal cord of TG-16 and TG-9 pigs. (B) Double immunofluorescence staining of the lumbar spinal cord of TG-16 pig revealing the colocalization of the nuclear hSOD1 inclusions with ubiquitin. (C) The nuclear inclusions are present in the spinal cord of transgenic hSOD1, but not WT, pig. We examined 677 neurons in three L4 spinal cord slices. (D, E) Immunofluorescent staining showing the presence of nuclear inclusions in the spinal cord (D) and cortex (E) of hSOD1 transgenic pig. Scale bars, A, B, D, and E, $50 \mu \mathrm{m} ; \mathbf{C}, 100 \mu \mathrm{m}$. (F) Spinal cord (SC) and brain (B) cortex were isolated from TG-16 and TG-9 transgenic pigs and lysed in RIPA buffer (left panel) or $2 \%$ SDS buffer (right panel). Western blotting with antibodies to hSOD1 or pan SOD1 shows that RIPA-treated lysates only display hSOD1 monomer bands, but 2\% SDS-treated lysates yielded hSOD1 oligomers. TG-16 pig had more hSOD1 oligomers than TG-9 pig. (G) Anti-ubiquitin probing the blot showing an increase in protein ubiquitination in the SC and brain cortex (B) from TG-16 pig. 


\section{Discussion}

Using nuclear transfer, we have established cloned pigs that express mutant hSOD1 protein with the ALSassociated SOD1 mutation G93A. Pathological and behavioral studies revealed that these transgenic pigs develop a hind limb motor disorder in association with motor neuron death, which is accompanied by astrogliosis and microgliosis, as well as skeletal muscle atrophy. The hSOD1 transgenic pigs showed disease characteristics typical of human ALS and unique nuclear inclusions that have not been seen in other animal models of ALS. Furthermore, we were able to establish F1 hSOD1 transgen- ic pigs that show germline transmissible expression of mutant hSOD1 and motor deficits. Thus, our transgenic pigs would provide a useful model for investigating the pathogenesis of ALS and identifying potential therapeutic targets.

It is well known that only mutant SOD1 forms aggregates, but overexpression of wild-type human SOD1 can also cause pathology in some transgenic mouse models [38]. Thus, it would be important to have a control pig model that expresses transgenic wild-type human SOD1 to rule out the non-specific effects of overexpressed transgenic proteins. Due to the cost in generating transgenic pigs and difficulty in controlling the expression of

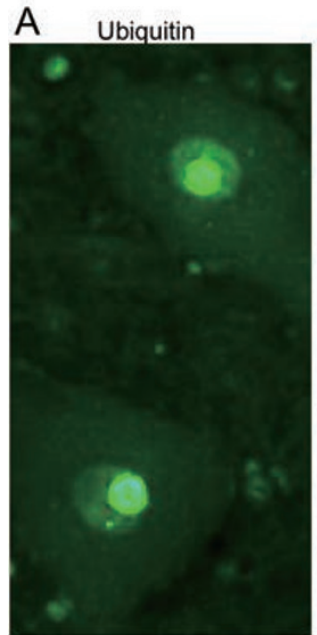

B Ubiquitin
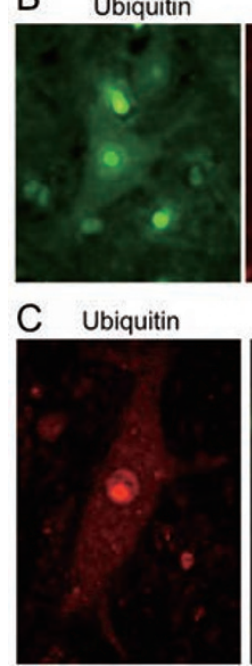

CHAT

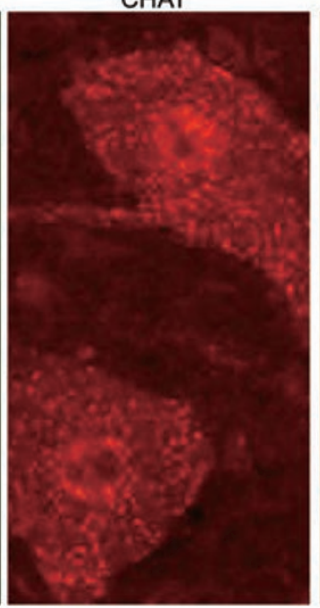

Calretinin

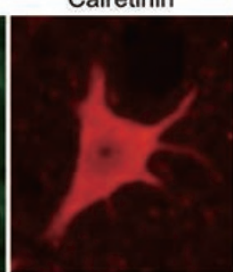

Parvalbumin

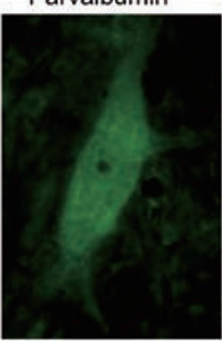

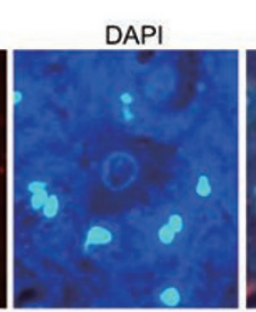

DAPI

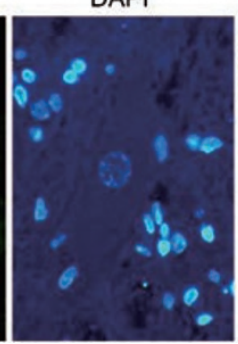

Merged

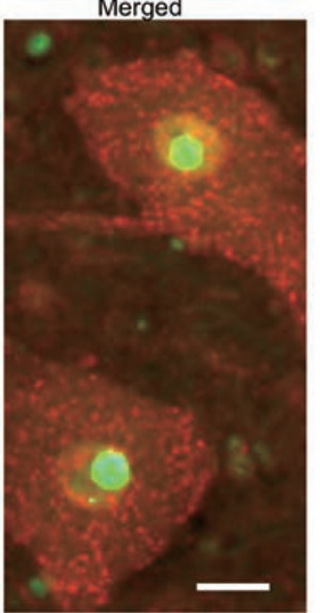

Merged

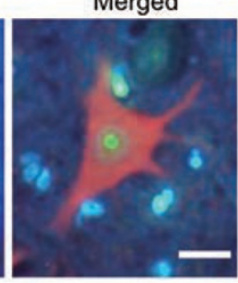

Merger

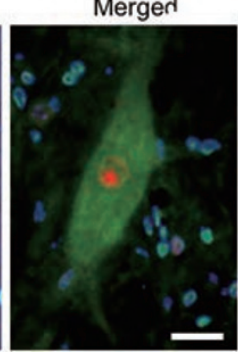

D

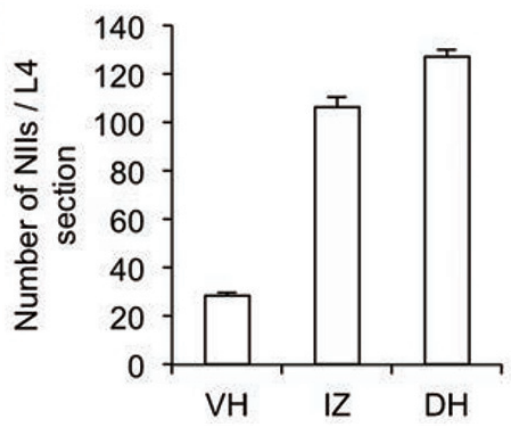

E

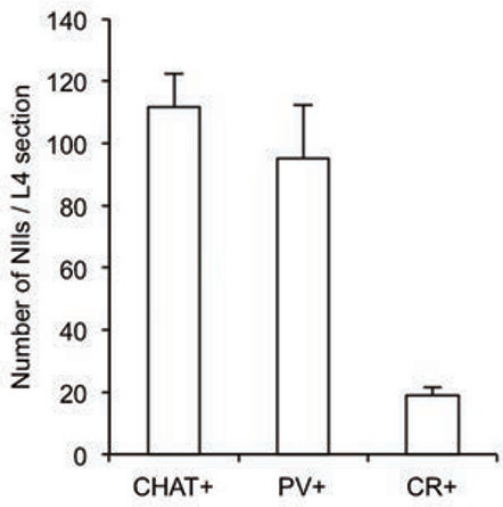

Figure 7 Distribution of nuclear inclusions (NIIs) in the lumbar spinal cord of TG-16 transgenic pig. (A-C) NIls are located in the (A) ChAT-, (B) CR-, and (C) Parvalbumin-positive neurons. (D) Quantitation of NIls in the L4 spinal cord showing that more NIIs are distributed in the dorsal horn $(\mathrm{DH})$ and intermediate zone (IZ) than ventral horn (VH). (E) Quantitation of the distribution of NIIs in ChAT-, CR-, and Parvalbumin-positive neurons, respectively. Data shown are means \pm SEM of three L4 slices. Scale bars, $20 \mu \mathrm{m}$. 

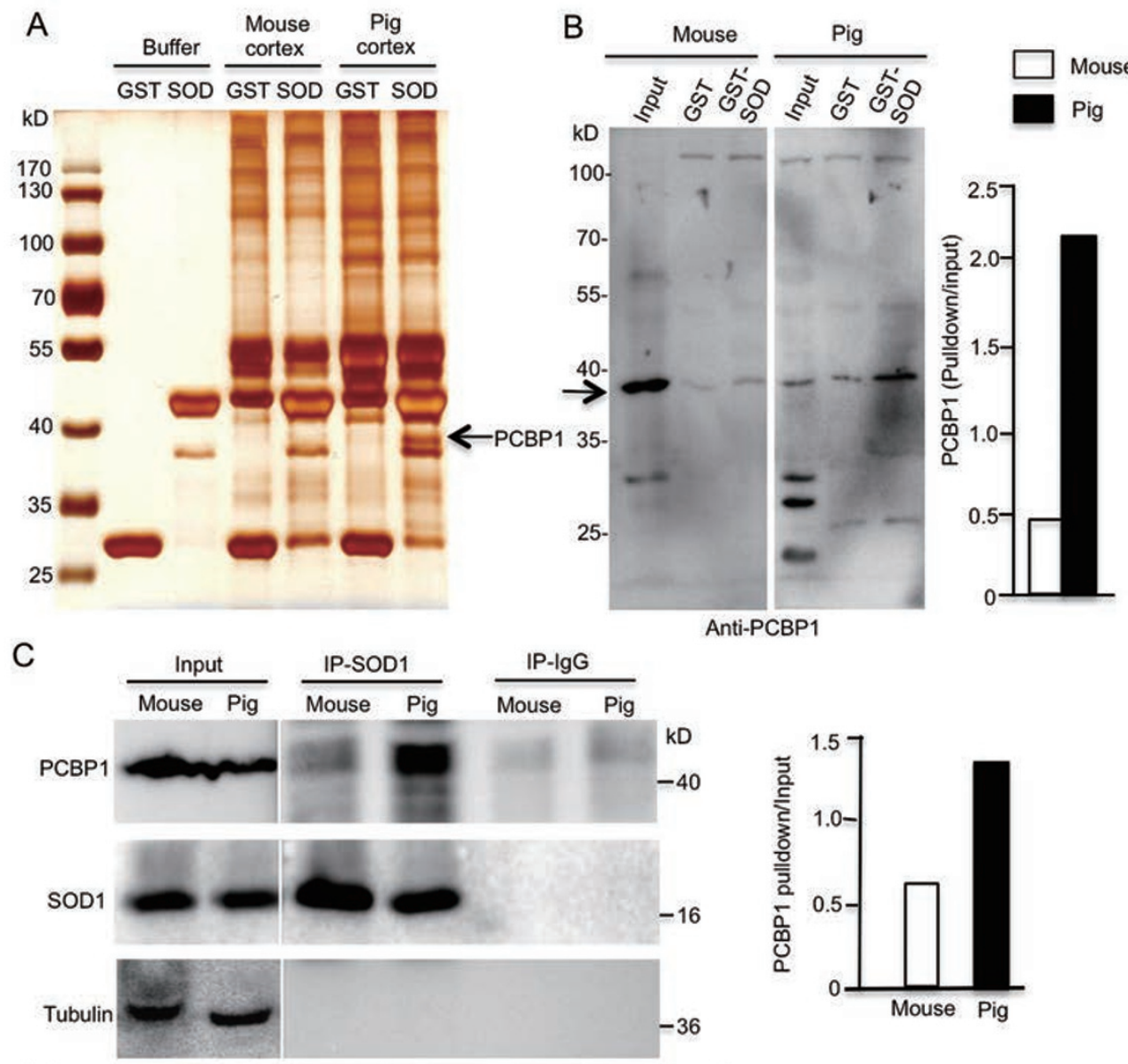

D

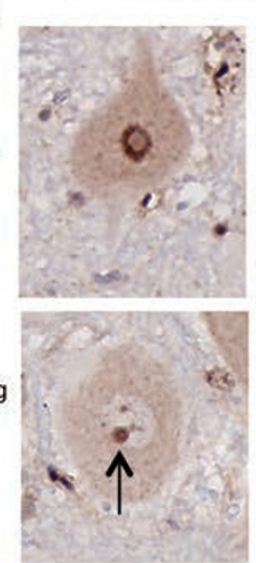

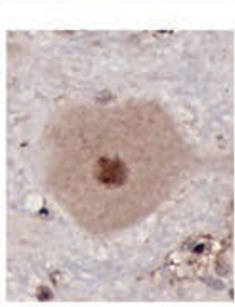

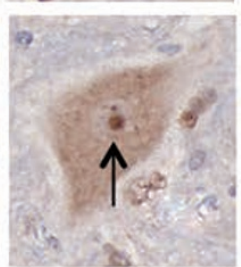

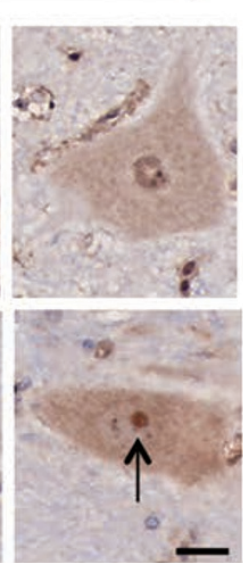

$E$
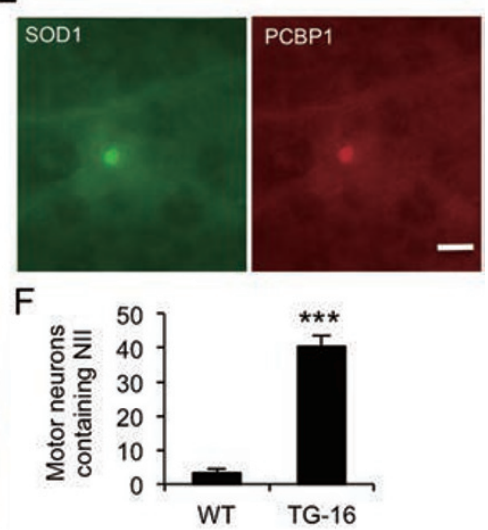

Figure 8 SOD1 interacts with PCBP1 in the pig brain. (A) GST-hSOD1 was used to pull down proteins from mouse and pig brain cortex tissue lysates. The pull-downs were analyzed by SDS PAGE. Silver staining revealed a band (arrow) specific to the pull-down from the pig brain tissue. Mass spectrometry identified the presence of PCBP1 in the band. (B) Western blot analysis with anti-PCBP1 verified that GST-hSOD1 binds more PCBP1 in the pig brain cortex lysates. The ratio of PCBP1 in pull-down to the input is presented (right panel). (C) Immunoprecipitation of SOD1 from mouse and pig brain cortex showing that more PCBP1 was coprecipitated with SOD1 in the pig brain tissue than in the mouse brain tissue. The ratios of precipitated proteins to input are also shown (right panel). (D) Immunostaining of the spinal cord of WT and hSOD1 transgenic pigs. While PCBP1 is diffuse in WT motor neurons, it is distributed in nuclear inclusions (arrows) in transgenic hSOD1 pig motor neurons. (E) Double immunofluorescent staining showing that both transgenic hSOD1 and endogenous PCBP1 are localized in the nuclear inclusions in the spinal cord. (F) Quantitation of the numbers of motor neurons containing PCBP1 nuclear inclusions in the spinal cord of WT and TG-16 transgenic pigs. Data are the mean \pm SEM of 3 L4 slices per group. ${ }^{* * *} P<0.001$ compared with WT. Scale bar, $20 \mu \mathrm{m}$. 
transgene, our studies did not include this control transgenic pig model. Despite this limitation, several lines of evidence support the idea that expression of mutant SOD1 caused the phenotypes of our hSOD1 transgenic pigs. First, western blotting results show that the expression levels of transgenic hSOD1 are lower than or equivalent to that of the endogenous pig SOD1, suggesting that transgenic hSOD1 is not overexpressed. Second, multiple transgenic pig founders show the similar limb movement impairment, and the levels of transgenic hSOD1 in general correlate with the severity of this phenotype. The phenotypes of our transgenic pigs are apparently correlated with the levels of misfolded SOD1. Higher expression or rapid accumulation of mutant SOD1 in motor neurons of the spinal cord usually induces a more severe disease phenotype, which was confirmed by the more severe phenotype and neurodegeneration in the TG-16 pig with more abundant misfolded hSOD1. This dose- and age-dependent phenomenon indicates that the accumulation of misfolded proteins with age is important for the development of ALS.

While the neurodegeneration, muscle atrophy, and limb movement deficits in our hSOD1 transgenic pigs resemble the pathological features seen in ALS patients and other animal models, we did obtain some novel findings from this large animal model. First, we found that transgenic pigs developed obvious limb movement deficits without showing cytoplasmic inclusions. The neuropathology of ALS is characterized by the presence of various forms of inclusion bodies in degenerating neurons and surrounding reactive astrocytes [39]. "Skeinlike inclusions" (SLIs) $[40,41]$ and Bunina bodies (BBs), which are cystatin $\mathrm{C}$-containing inclusions in the cell bodies [42, 43], are typical inclusions found in motor neurons in ALS. Tar DNA-binding protein 43 (TDP-43) is also localized in cytoplasmic ubiquitinated inclusions in sALS patient brains. We did not find these inclusions in our hSOD1 transgenic pig brains and spinal cords. Ubiquitin-positive SLIs are known to be characteristic features of adult-onset ALS [44], and there is no relation between ALS with BBs [45]. Also, TDP-43 is absent in the inclusions in fALS caused by SOD1 mutations [46]. Because our hSOD1 transgenic pigs do not overexpress mutant hSOD1 and have only been alive for less than 2 years, they are more likely to be at the early disease stage. The findings from these transgenic pigs indicate that early ALS symptoms associated with SOD1 mutations can occur in the absence of cytoplasmic SOD1 aggregates.

Importantly, we found the nuclear accumulation of mutant SOD1 in hSOD1 transgenic pig brain and spinal cord. Nuclear inclusions are more prominent in motor neurons in the spinal cord of hSOD1 transgenic pigs. In fact, ubiquitin-positive inclusions have been reported in some cases of human ALS with SOD1 mutations, but have not ever been found in transgenic SOD1 mouse models $[21,22]$. Ubiquitinated nuclear inclusions are the common pathological hallmark of most polyglutamine diseases, such as Huntington's disease and spinocerebellar ataxia [47]. Colocalization of ubiquitin with transgenic hSOD1 in nuclear inclusions is evidence for the pathological accumulation of transgenic hSOD1 in the pig brain, suggesting that the nuclear SOD1 inclusions, like other misfolded protein inclusions, share the same biochemical processes involving the ubiquitin-proteasome system.

Although we do not yet know whether ubiquitinated inclusions have direct toxicity in transgenic pig neurons, the nuclear inclusions should result from the accumulation of transgenic mutant hSOD1. The presence of these inclusions in motor neurons is consistent with motor neuronal degeneration and associated with limb movement defects. Although transgenic SOD1 mouse models do not show such nuclear SOD1 inclusions, fractionation of the nucleus of transgenic SOD1 mouse brain demonstrates the presence of wild-type and transgenic SOD1 in the nucleus at relatively low levels [48]. Because SOD1 is a small protein, it can passively diffuse into the nucleus or be shuttled freely between the nucleus and cytoplasm [49]. These facts raise the important issue of whether pig brains contain proteins that can interact with mutant hSOD1 to keep it in the nucleus or modulate hSOD1 activity for its nuclear accumulation. Using GST pulldown and mass spectrometry, we identified PCBP1 as a partner for binding to hSOD1 in the pig brain, but not in mouse brain. PCBP1 is a nuclear protein and, along with PCBP-2 and hnRNPK, corresponds to the major cellular poly(rC)-binding protein and has widely diversified functions in mRNA binding and stabilization, translational activation or silencing [35, 37, 50], as well as iron chaperone function [36]. Since most sALS cases harbor cytoplasmic inclusions containing TDP-43, nuclear proteins that are involved in binding DNA and RNA, nuclear dysfunction and abnormal processing of DNA and RNA are thought to play a role in ALS pathogenesis [51]. Although we did not find TDP-43 inclusions in our transgenic pig models, the nuclear localization of mutant hSOD1 suggests that fALS may also have impaired nuclear function, at least in the early stage of disease.

The presence of nuclear inclusions in hSOD1 transgenic pigs and the interaction of SOD1 with PCBP1 are interesting findings in our studies. While nuclear inclusions have been found in some adult ALS patient brains, whether they are also present in ALS patients at young 
ages requires further studies to verify. The interaction of hSOD1 with PCBP1 explains why hSOD1 transgenic pigs exhibit the nuclear accumulation of mutant hSOD1. Species-specific partners or targets may be important for neurodegenerative diseases in large mammals as well as humans. The difference in the nuclear inclusions between transgenic SOD1 rodent and pig models suggests that species differences may modulate the distribution and aggregation of mutant SOD1. Because mutant SOD1 binds more PCBP1, this effect could impair the function of PCBP1, and with other toxic gain of function, contributes to ALS neuropathology. The nuclear accumulation of mutant hSOD1 could also affect nuclear function, and such nuclear dysfunction can lead to ALS-related neurological symptoms in the absence of cytoplasmic inclusions during the early stages of disease. This also has important implications for identifying an effective therapeutic target, as preventing early neuropathological events is the best way to treat ALS and other protein misfolding-related diseases.

\section{Materials and Methods}

\section{Animals}

Animal use followed the NIH Guide for the Care and Use of Laboratory Animals. The animal use protocol was approved by the Institutional Animal Care and Use Committees at Guangzhou Institute of Biomedicine and Health, Chinese Academy of Sciences.

\section{Production of hSODl (G93A) transgenic pigs}

The cDNA of human SOD1 carrying a $\mathrm{G} \rightarrow \mathrm{C}$ mutation at the position of $281 \mathrm{bp}$, which substitutes glutamine with alanine (G93A), was ligated to the $\mathrm{Sal} \mathrm{I} / \mathrm{Not} \mathrm{I}$ sites of the plasmid $p E G F P$ $N 1$ (Clontech, Palo Alto, CA, USA) to generate a vector [ $p C M V$ hSOD1(G93A)]. The final construct was confirmed by sequence analysis. Primary pig fetal fibroblasts were isolated from a 35day fetus of a female Tibet miniature pig. The fetal fibroblasts were cultured in Dulbecco's modified Eagle's medium (DMEM, HyClone, Logan, UT, USA) supplemented with $15 \%$ fetal bovine serum (FBS, HyClone, Logan, UT, USA) and 1\% (v:v) penicillin/ streptomycin $(10000 \mathrm{U} / \mathrm{ml}$ penicillin, $10000 \mu \mathrm{g} / \mathrm{ml}$ streptomycin; GIBCO-BRL, Grand Island, NY, USA) at $39{ }^{\circ} \mathrm{C}$ in an incubator with $5 \% \mathrm{CO}_{2} . p C M V-h S O D 1(G 93 A)$ was linearized with $A p a L \mathrm{I}$ and transfected into the fibroblasts by electroporation (Gene Pulser Xcell, Bio-Rad, Hercules, CA, USA). The cells were split 1:10 into fresh culture medium after transfection. After $48 \mathrm{~h}, 800 \mu \mathrm{g} / \mathrm{ml}$ G418 (Merck, Darmstadt, Germany) was added to the medium to select transfected cell colonies, and the plates were incubated in media containing G418 for $\sim 10$ days. The surviving cell colonies were selected and propagated in a fresh 48 -well plate. Colonies that proliferated well were then expanded and screened for the expression of hSOD1. The positive colonies were frozen in small aliquots. Prior to SCNT, hSOD1-expressing transfected cells were thawed and cultured until they reached sub-confluence.

The ovaries of pigs were collected from long white pigs at a local slaughterhouse and transported to the laboratory in $0.9 \%$
$\mathrm{NaCl}$ at $30{ }^{\circ} \mathrm{C}-35{ }^{\circ} \mathrm{C}$. The cumulus-oocyte complexes (COCs) were aspirated from the antral follicles and washed three times in maturation medium, which contained tissue culture medium 199 (TCM-199, 31100-035; Gibco-BRL, Grand Island, NY, USA) supplemented with $0.1 \%(\mathrm{w} / \mathrm{v})$ polyvinyl alcohol (P8136; Sigma, St. Louis, MO, USA), 3.05 mM D-glucose (G7528; Sigma), 0.91 mM sodium pyruvate (P4562; Sigma), 0.57 mM L-cysteine (C7352; Sigma), $0.5 \mu \mathrm{g} / \mathrm{ml}$ of luteinizing hormone (L5269; Sigma), 0.5 $\mu \mathrm{g} / \mathrm{ml}$ of follicle-stimulating hormone (F2293; Sigma), $10 \mathrm{ng} / \mathrm{ml}$ of epidermal growth factor (E4127; Sigma), $10 \%(\mathrm{v}: \mathrm{v})$ of porcine follicular fluid, $75 \mu \mathrm{g} / \mathrm{ml}$ of penicillin $\mathrm{G}$, and $50 \mu \mathrm{g} / \mathrm{ml}$ of streptomycin. The oocytes were matured for $40 \mathrm{~h}$ at $39^{\circ} \mathrm{C}$ in an incubator containing $5 \% \mathrm{CO}_{2}$. Fifty to sixty COCs were transferred to $500 \mu \mathrm{l}$ of maturation medium that had been covered with mineral oil in a 4-well multi-dish (Nunc, Roskilde, Denmark) and pre-equilibrated at $39{ }^{\circ} \mathrm{C}$ in an atmosphere of $5 \% \mathrm{CO}_{2}$ overnight. After $42-44 \mathrm{~h}$ of culture, oocytes were separated from cumulus cells by vigorous vortex for $4 \mathrm{~min}$ in TL-Hepes supplemented with $0.1 \%$ polyvinyl alcohol (P8136; Sigma) and 0.1\% hyaluronidase (H3506; Sigma). Denuded mature oocytes were enucleated by removal of the polar body and the associated metaphase plate using a 30- $\mu \mathrm{m}$ diameter beveled glass pipette in HEPES-buffered TCM-199 supplemented with $0.3 \%$ BSA (A8022; Sigma) and $7.5 \mu \mathrm{g} / \mathrm{ml}$ cytochalasin B (C6762; Sigma). A single donor cell was injected into the perivitelline space of the oocyte in contact with the oocyte membrane.

Injected oocyte-fibroblast pairs were placed between two $0.2 \mathrm{~mm}$ diameter platinum electrodes kept $1 \mathrm{~mm}$ apart in fusion and activation medium in a position vertical to the electrodes. Fusion and activation were induced simultaneously with two successive DC pulses of $1.1 \mathrm{kV} / \mathrm{cm}$ for $30 \mathrm{~ms}$ using an Electro Cell Manipulator 200 (Genetronics, San Diego, CA, USA). After fusion and activation, embryos were kept in micromanipulation medium without $\mathrm{CB}$ for an additional $30 \mathrm{~min}$ before the fusion rate was evaluated. Reconstructed embryos (50-100) were transferred to $500 \mu \mathrm{l}$ of culture medium covered with mineral oil in a 4-well multi-dish and cultured for $18-22 \mathrm{~h}$ at $39{ }^{\circ} \mathrm{C}$ in an atmosphere of $5 \% \mathrm{CO}_{2}$. The embryos were then surgically transferred into the oviduct of a surrogate female on the first day of standing estrus. Pregnancy status was monitored using an ultrasound scanner between days 30-35 post-transplantation. Some embryos were cultured for 6 days to test the blastocyst formation rate, as well as developmental ability. Transgenic piglets were identified by PCR analysis of ear DNA with the following primers: SOD-F: 5'-ATGCGGTTTTGGCAGTACAT-3'; SOD-R: 5'-TATTGGGCGATCCCAATTAC-3'.

\section{Histology, immunohistochemistry, and immunofluorescence staining}

The tissues (lumbar spinal cord, brain cortex, gastrocnemius, soleus, and tibialis anterior muscles) were fixed in situ with $4 \%$ buffered paraformaldehyde for $48 \mathrm{~h}$. The entire tissue was then dissected, embedded in paraffin wax, and exhaustively crosssectioned at $3 \mu \mathrm{M}$ to a slide. Sections were deparaffinized in xylene and rehydrated in a graded series of alcohol, followed by $\mathrm{dH}_{2} \mathrm{O}$, stained with cresyl violet or with hematoxylin and eosin, differentiated, and coverslipped. For immunohistochemistry and immunofluorescence staining, antigen retrieval was performed in a $1 \mathrm{M}$ citrate buffer $(\mathrm{pH}=6.0)$ bath for $20 \mathrm{~min}$. Tissues were immunostained with primary antibodies and visualized using an ammonium bicarbonate (ABC) Kit in combination with diami- 
nobenzidine (Vector Laboratories, Burlingame, CA, USA). Some immunostained sections were counterstained with hematoxylin to display nuclei. Double-labeled immunofluorescence staining was used to examine the colocalization of ubiquitin with hSOD1, PV, CB or ChAT and hSOD1 with PCBP1. Tissue sections were incubated first with specific primary antibodies, and then with secondary antibodies labeled with fluorescent dyes. DAPI was used to label nuclear DNA.

\section{Morphometric analysis and statistics}

Quantitative analyses of motor neurons were counted on serial lumbar 4 (L4) sections immunoperoxidase-stained for ChAT and NeuN. Motor neurons were defined as large ChAT-IR neurons (diameter $\geq 25 \mu \mathrm{m}$ ) located in the motor columns (Rexed's laminae IX). For anti-NeuN staining, large NeuN-IR neurons $(\geq 25 \mu \mathrm{m})$ in the laminae IX were considered as motor neurons. Neurons with smaller diameter $(<25 \mu \mathrm{m})$ in the other laminae were termed interneurons. For quantitating the number and distribution of NIIs in lumbar spinal cord, sections were immunostained using an ubiquitin antibody as described above, and the number of NIIs was counted in half of the lumbar spinal cord. At least three cross-sections were counted for each spinal cord. Furthermore, we counted the numbers of costained neuron cells in sections double labeled with ubiquitin/PV, ubiquitin/CR, and ubiquitin/ChAT, respectively.

\section{Electron microscopy (EM)}

For EM examination of pig spinal cord morphology, postmortem pig lumbar spinal cords that were removed within $2-3 \mathrm{~h}$ after death were fixed for $72 \mathrm{~h}$ in $4 \%$ paraformaldehyde $/ 2.5 \%$ glutaradehyde/0.1 M PB. The sections were then fixed in $1 \%$ buffered osmium tetroxide, dehydrated with ethanol, and embedded in Eponate 12 resin (Ted Pella Inc., Redding, CA, USA). Ultrathin sections were cut on a Leica UC6rt ultramicrotome (Leica Microsystems, Bannockburn, IL, USA) at 70-80 nm, placed on Formvar-coated grids, and counter-stained with $4 \%$ aqueous uranyl acetate and $2 \%$ lead citrate. Sections were examined using a Hitachi H-7500 transmission electron microscope (Hitachi High Technologies of America, Inc., Pleasanton, CA, USA) equipped with a Gatan BioScan CCD camera.

\section{Behavioral tasks}

The movement capabilities of transgenic pigs were tested by treadmill running. A suitable cage was placed onto the treadmill to fix the pig and make it run on the conveyer belt. Two methods were used to test and quantify the running ability of pigs on the treadmill. The first was the accelerating speed mode, in which the speed of the treadmill accelerated from 1.0 to $6.0 \mathrm{~km} / \mathrm{h}$ gradually when the pig ran on the treadmill. Each speed was maintained for $10 \mathrm{~s}$ until $6.0 \mathrm{~km} / \mathrm{h}$. The maximal speed at which the pig could not run appropriately or fluently (such as sit, lie down, lean on the back wall of the cage) was recorded. The second method was called the fixed speed mode, in which the speed of treadmill was kept at $3.0 \mathrm{~km} / \mathrm{h}$ for $1 \mathrm{~min}$ when the pig ran on it. The better running time was recorded as the efficient running time. The treadmill running test was performed once a month after the pigs were grown. Non-transgenic littermates or age- and body weightmatched wild-type pigs were used as controls.

\section{$E M G$}

Transgenic and wild-type pigs were measured by quantitative EMG, three muscles were tested: the bilateral tibialis anterior muscles and the right sternocleidomastoid muscle. All of the EMG examinations were performed by one person, who is an experienced electromyographer. The EMG test was performed on the Keypoint ${ }^{\circledR}$ EMG equipment (Medtronic Dantec, Copenhagen, Denmark) provided with specific software for data acquisition and processing at the same time and immediately after needle EMG on the same test session. Spontaneous activity (fibrillation potentials and positive sharp-waves were observed) at rest was recorded at several insertions outside the end-plate regions in muscles, and denervation activity at more than two sites examined in the muscle was considered abnormal. Motor unit potentials (MUPs) recorded at weak effort were quantified offline with measurements of durations, amplitudes, and multiphase waves ( $\geq 4)$ of at least 20 MUPs. We used two transgenic pigs and two age-matched WT pigs for EMG analysis. Every pig has three muscle samples tested (left and right tibialis anterior muscles as well as right sternocleidomastoid muscles). MUP data were recorded, and many wave photos were recorded in the monitor screen. Each wave is a MUP. We selected 20 waves of each muscle from each pig for measuring its duration, amplitude, and polyphasia and obtained a mean value of these waves. The recruitment order was measured during maximal voluntary contraction (MVC) of tested muscle. The phase and amplitude of electrical activity during MVC in muscles were recorded. The following test settings were used: the sensitivity is $50 \mu \mathrm{V} /$ day and the scanning speed is $5 \mathrm{~ms} /$ day for spontaneous activity, 0.2 $\mathrm{mV} /$ day, and $10 \mathrm{~ms} /$ day for MUPs, $0.5 \mathrm{mV} /$ day, $100 \mathrm{~ms} /$ day for MVC; filters $20 \mathrm{~Hz}$ to $10 \mathrm{kHz}$.

\section{GST pull-down assay and mass spectrometric analysis}

GST fusion protein containing hSOD1 was generated and purified by glutathione resin with $1 \times$ PBS equilibration buffer and $10 \mathrm{mM}$ reduced glutathione elution buffer. Cerebral cortex lysates of pig or mouse $(0.1 \mathrm{~g} / \mathrm{ml})$ in NP-40 buffer $(50 \mathrm{mM}$ Tris $\mathrm{pH} 7.4,50$ $\mathrm{mM} \mathrm{NaCl}, 0.1 \%$ Triton X-100, $1 \%$ NP40) were homogenized using a Teflon homogenizer for 30 strokes, sonicated for $10 \mathrm{~s}$ on ice, and centrifuged at $1000 \times g$ at $4{ }^{\circ} \mathrm{C}$ for $3 \mathrm{~min}$. The supernatants $(5$ $\mu \mathrm{g} / \mu 1$ protein) in a $1.5-\mathrm{ml}$ tube were incubated with GST or GSThSOD1 $(100 \mu \mathrm{g})$ and $50 \mu \mathrm{l}$ glutathione beads at $4{ }^{\circ} \mathrm{C}$ for $3 \mathrm{~h}$. The precipitates were subjected to SDS PAGE or western blotting. The gel containing interesting bands was decolored by $25 \mathrm{mM} \mathrm{ABC}$, $50 \%$ acrylonitrile (ACN), and dehydrated by $100 \%$ ACN. Dehydrated gel was deoxidized at $56{ }^{\circ} \mathrm{C}$ for $1 \mathrm{~h}$ with $10 \mathrm{mM}$ DTT, 50 $\mathrm{mM} \mathrm{ABC}$ buffer and incubated with $55 \mathrm{mM}$ Indole-3-acetic acid for neutralizing the remainder of the DTT at room temperature in the dark. The gel containing interesting proteins was digested by $10 \mathrm{ng} / \mathrm{ml}$ trypsin at $37{ }^{\circ} \mathrm{C}$ overnight, extracted by $50 \% \mathrm{ACN}$ and $5 \%$ Trifluoroacetic acid (TFA) two times, and then freeze-dried. The freeze-drying gel materials were dissolved by $0.1 \%$ TFA and analyzed by AB SCIEX TripleTOF ${ }^{\circledR} 5600$ system for mass spectrometry analysis.

\section{Statistical analysis}

Statistical analysis was performed with SPSS software (SPSS, Inc., Chicago, IL, USA). Mean values were analyzed for significance using a Student's $t$-test or ANOVA as described in the text. The difference in the mean values between the two groups was considered significant for a $P$-value $<0.05$. 
Detailed methods are described in the Supplementary information, Data S1.

\section{Acknowledgments}

This work was supported by the National Basic Research Program of China (973 program, 2011CB944203), the National Natural Science Foundation of China (31071293) to LL; the National Institutes of Health (NS036232, NS041669 and NS045016) to XL and SL; Talent Program of Yunnan Province, China, and The Professorial Fellowship of Monash University, Australia to ZX; the National Natural Science Foundation of China (81171179, 81272439), Key Sci-Tech Research Projects of Guangdong Province, China (2008A030201019) to XJ, and The State Key Laboratory of Molecular Developmental Biology, China. We would like to thank the staffs of the pig farm for animal husbandry and assistance in the behavioral tasks. We thank Cheryl Strauss for critically reading this manuscript.

\section{References}

1 Dion PA, Daoud H, Rouleau GA. Genetics of motor neuron disorders: new insights into pathogenic mechanisms. Nat Rev Genet 2009; 10:769-782.

2 Boillée S, Vande Velde C, Cleveland DW. ALS: a disease of motor neurons and their nonneuronal neighbors. Neuron 2006; 52:39-59.

3 Rosen DR, Siddique T, Patterson D, et al. Mutations in $\mathrm{Cu} / \mathrm{Zn}$ superoxide dismutase gene are associated with familial amyotrophic lateral sclerosis. Nature 1993; 362:59-62.

4 Turner BJ, Talbot K. Transgenics, toxicity and therapeutics in rodent models of mutant SOD1-mediated familial ALS. Prog Neurobiol 2008; 85:94-134.

5 Gurney ME, Pu H, Chiu AY, et al. Motor neuron degeneration in mice that express a human $\mathrm{Cu}, \mathrm{Zn}$ superoxide dismutase mutation. Science 1994; 264:1772-1775.

6 Reaume AG, Elliott JL, Hoffman EK, et al. Motor neurons in $\mathrm{Cu} / \mathrm{Zn}$ superoxide dismutase-deficient mice develop normally but exhibit enhanced cell death after axonal injury. Nat Genet 1996; 13:43-47.

7 Julien JP. Amyotrophic lateral sclerosis. unfolding the toxicity of the misfolded. Cell 2001; 104:581-591.

8 Bendotti C, Carrì MT. Lessons from models of SOD1-linked familial ALS. Trends Mol Med 2004; 10:393-400.

9 Ferraiuolo L, Kirby J, Grierson AJ, Sendtner M, Shaw PJ. Molecular pathways of motor neuron injury in amyotrophic lateral sclerosis. Nat Rev Neurol 2011; 7:616-630.

10 Martin LJ, Liu Z, Chen K, et al. Motor neuron degeneration in amyotrophic lateral sclerosis mutant superoxide dismutase-1 transgenic mice: mechanisms of mitochondriopathy and cell death. J Comp Neurol 2007; 500:20-46.

11 Dal Canto MC, Gurney ME. Neuropathological changes in two lines of mice carrying a transgene for mutant human $\mathrm{Cu}, \mathrm{Zn} \mathrm{SOD}$, and in mice overexpressing wild type human SOD: a model of familial amyotrophic lateral sclerosis (FALS). Brain Res 1995; 676:25-40.

12 Shibata N. Transgenic mouse model for familial amyotrophic lateral sclerosis with superoxide dismutase-1 mutation. $\mathrm{Neu}$ ropathology 2001; 21:82-92.
13 Wong PC, Pardo CA, Borchelt DR, et al. An adverse property of a familial ALS-linked SOD1 mutation causes motor neuron disease characterized by vacuolar degeneration of mitochondria. Neuron 1995; 14:1105-1116.

14 Guegan C, Przedborski S. Programmed cell death in amyotrophic lateral sclerosis. J Clin Invest 2003; 111:153-161.

15 Wengenack TM, Holasek SS, Montano CM, Gregor D, Curran GL, Poduslo JF. Activation of programmed cell death markers in ventral horn motor neurons during early presymptomatic stages of amyotrophic lateral sclerosis in a transgenic mouse model. Brain Res 2004; 1027:73-86.

16 Reyes NA, Fisher JK, Austgen K, VandenBerg S, Huang EJ, Oakes SA. Blocking the mitochondrial apoptotic pathway preserves motor neuron viability and function in a mouse model of amyotrophic lateral sclerosis. J Clin Invest 2010; 120:3673-3679.

17 Honig LS, Rosenberg RN. Apoptosis and neurologic disease. Am J Med 2000; 108:317-330.

18 Migheli A, Atzori C, Piva R, et al. Lack of apoptosis in mice with ALS. Nat Med 1999; 5:966-967.

19 He BP, Strong MJ. Motor neuronal death in sporadic amyotrophic lateral sclerosis (ALS) is not apoptotic. A comparative study of ALS and chronic aluminium chloride neurotoxicity in New Zealand white rabbits. Neuropathol Appl Neurobiol 2000; 26:150-160.

20 Embacher N, Kaufmann WA, Beer R, et al. Apoptosis signals in sporadic amyotrophic lateral sclerosis: an immunocytochemical study. Acta Neuropathol 2001; 102:426-434.

21 Kakita A, Oyanagi K, Nagai H, Takahashi H. Eosinophilic intranuclear inclusions in the hippocampal pyramidal neurons of a patient with amyotrophic lateral sclerosis. Acta Neuropathol 1997; 93:532-536.

22 Seilhean D, Takahashi J, El Hachimi KH, et al. Amyotrophic lateral sclerosis with neuronal intranuclear protein inclusions. Acta Neuropathol 2004; 108:81-87.

23 Forsberg K, Andersen PM, Marklund SL, Brännström T. Glial nuclear aggregates of superoxide dismutase- 1 are regularly present in patients with amyotrophic lateral sclerosis. Acta Neuropathol 2011; 121:623-634.

24 Prather RS, Hawley RJ, Carter DB, Lai L, Greenstein JL. Transgenic swine for biomedicine and agriculture. Theriogenology 2003; 59:115-123.

25 Aigner B, Renner S, Kessler B, et al. Transgenic pigs as models for translational biomedical research. J Mol Med (Berl) 2010; 88:653-664.

26 Yang D, Yang H, Li W, et al. Generation of PPAR $\gamma$ monoallelic knockout pigs via zinc-finger nucleases and nuclear transfer cloning. Cell Res 2011; 21:979-982.

27 Yang D, Wang CE, Zhao B, et al. Expression of Huntington's disease protein results in apoptotic neurons in the brains of cloned transgenic pigs. Hum Mol Genet 2010; 19:3983-3994.

28 Gordon T, Ly V, Hegedus J, Tyreman N. Early detection of denervated muscle fibers in hindlimb muscles after sciatic nerve transection in wild type mice and in the G93A mouse model of amyotrophic lateral sclerosis. Neurol Res 2009; 31:28-42.

29 Gosztonyi G, Naschold U, Grozdanovic Z, StoltenburgDidinger G, Gossrau R. Expression of Leu-19 (CD56, N$\mathrm{CAM}$ ) and nitric oxide synthase (NOS) $\mathrm{I}$ in denervated and 
reinnervated human skeletal muscle. Microsc Res Tech 2001; 55:187-197.

30 Dupuis L, Gonzalez de Aguilar JL, di Scala F, M, et al. Nogo provides a molecular marker for diagnosis of amyotrophic lateral sclerosis. Neurobiol Dis 2002; 10:358-365.

31 Atkin JD, Scott RL, West JM, Lopes E, Quah AK, Cheema SS. Properties of slow- and fast-twitch muscle fibres in a mouse model of amyotrophic lateral sclerosis. Neuromuscul Disord 2005; 15:377-388.

32 de Carvalho M, Dengler R, Eisen A, et al. Electrodiagnostic criteria for diagnosis of ALS. Clin Neurophysiol 2008; 119:497-503.

33 Okamoto K, Hirai S, Amari M, Sakurai A. Electron micrograph of ubiquitin-positive intraneuronal inclusions in the extra-motor cortices in patients with amyotrophic lateral sclerosis. Neuropathology 1996; 16:112-116.

34 Piao YS, Wakabayashi K, Kakita A, et al. Neuropathology with clinical correlations of sporadic amyotrophic lateral sclerosis: 102 autopsy cases examined between 1962 and 2000. Brain Pathol 2003; 13:10-22.

35 Makeyev AV, Liebhaber SA. The poly(C)-binding proteins: a multiplicity of functions and a search for mechanisms. RNA 2002; 8:265-278.

36 Nandal A, Ruiz JC, Subramanian P, et al. Activation of the HIF prolyl hydroxylase by the iron chaperones PCBP1 and PCBP2. Cell Metab 2011; 14:647-657.

37 Xia M, He H, Wang Y, et al. PCBP1 is required for maintenance of the transcriptionally silent state in fully grown mouse oocytes. Cell Cycle 2012; 11:2833-2842.

38 Jaarsma D, Haasdijk ED, Grashorn JA, et al. Human $\mathrm{Cu} / \mathrm{Zn}$ superoxide dismutase (SOD1) overexpression in mice causes mitochondrial vacuolization, axonal degeneration, and premature motoneuron death and accelerates motoneuron disease in mice expressing a familial amyotrophic lateral sclerosis mutant SOD1. Neurobiol Dis 2000; 7:623-643.

39 Barbeito LH, Pehar M, Cassina P, et al. A role for astrocytes in motor neuron loss in amyotrophic lateral sclerosis. Brain Res Brain Res Rev 2004; 47:263-274.

$40 \mathrm{He}$ CZ, Hays AP. Expression of peripherin in ubiquinated inclusions of amyotrophic lateral sclerosis. J Neurol Sci 2004; 217:47-54.

41 Kawashima T, Furuta A, Doh-ura K, Kikuchi H, Iwaki T.
Ubiquitin-immunoreactive skein-like inclusions in the neostriatum are not restricted to amyotrophic lateral sclerosis, but are rather aging-related structures. Acta Neuropathol 2000; 100:43-49.

42 Okamoto K, Hirai S, Amari M, Watanabe M, Sakurai A. Bunina bodies in amyotrophic lateral sclerosis immunostained with rabbit anti-cystatin C serum. Neurosci Lett 1993; 162:125-128.

43 Sasaki S, Maruyama S. Immunocytochemical and ultrastructural studies of the motor cortex in amyotrophic lateral sclerosis. Acta Neuropathol 1994; 87:578-585.

44 Matsumoto S, Goto S, Kusaka H, et al. Ubiquitin-positive inclusion in anterior horn cells in subgroups of motor neuron diseases: a comparative study of adult-onset amyotrophic lateral sclerosis, juvenile amyotrophic lateral sclerosis and Werdnig-Hoffmann disease. J Neurol Sci 1993; 115:208-213.

45 van Welsem ME, Hogenhuis JA, Meininger V, Metsaars WP, Hauw JJ, Seilhean D. The relationship between Bunina bodies, skein-like inclusions and neuronal loss in amyotrophic lateral sclerosis. Acta Neuropathol 2002; 103:583-589.

46 Maekawa S, Leigh PN, King A, et al. TDP-43 is consistently co-localized with ubiquitinated inclusions in sporadic and Guam amyotrophic lateral sclerosis but not in familial amyotrophic lateral sclerosis with and without SOD1 mutations. Neuropathology 2009; 29:672-683.

47 Everett CM, Wood NW. Trinucleotide repeats and neurodegenerative disease. Brain 2004; 127:2385-2405.

48 Gertz B, Wong M, Martin LJ. Nuclear localization of human SOD1 and mutant SOD1-specific disruption of survival motor neuron protein complex in transgenic amyotrophic lateral sclerosis mice. J Neuropathol Exp Neurol 2012; 71:162-177.

49 Chang LY, Slot JW, Geuze HJ, Crapo JD. Molecular immunocytochemistry of the $\mathrm{CuZn}$ superoxide dismutase in rat hepatocytes. J Cell Biol 1988; 107:2169-2179.

50 Pickering BM, Mitchell SA, Spriggs KA, Stoneley M, Willis AE. Bag-1 internal ribosome entry segment activity is promoted by structural changes mediated by poly(rC) binding protein 1 and recruitment of polypyrimidine tract binding protei n 1. Mol Cell Biol 2004; 24:5595-5605.

51 Chen-Plotkin AS, Lee VM, Trojanowski JQ. TAR DNA-binding protein 43 in neurodegenerative disease. Nat Rev Neurol 2010; 6:211-220.

(Supplementary information is linked to the online version of the paper on the Cell Research website.) 